\title{
11
}

\section{The Induction of Synaesthesia in Non-Synaesthetes}

\author{
Devin B. Terhune, David P. Luke, and Roi Cohen Kadosh
}

Abstract
In this chapter we review research examining the induction of synaesthesia with training,
posthypnotic suggestion, and pharmacological agents in non-synaesthetes. Each of these methods
has been shown to produce different aspects of synaesthesia, but none have produced
parallels between induced and congenital synaesthesias have the potential to illuminate different
facets of this condition. We argue that training may be a valuable model for studying the learning
pharmacological agents may serve as a useful tool for studying the development of synaesthesia
or for large-scale studies of induced synaesthesia. Induced synaesthesias also raise important
questions regarding espoused criteria for demarcating synaesthesia from other phenomena.




\subsection{Introduction}

Synaesthesia is an unusual but healthy neurological condition in which a stimulus, such as the number 7 or the note $E$, reliably and involuntarily elicits an atypical concurrent experience, such as the colour red (Ward, 2013). Synaesthesia occurs in a small minority of the population ( $\sim 4$ per cent; Simner et al., 2006) and has been shown to impact a wide range of cognitive and perceptual abilities from selective attention to episodic memory (Ward, 2013). In turn, uncovering the characteristics and mechanisms of this condition has the potential to inform our understanding of a diverse array of processes and functions including automaticity (Mattingley, 2009), conscious awareness (Cohen Kadosh and Henik, 2007), and memory (Rothen, Meier, and Ward, 2012).

Experimentally manipulating a phenomenon, such as by inducing, disrupting, or modulating it, can often yield information regarding both fundamental and ancillary characteristics as well as necessary and sufficient conditions for the expression of the phenomenon. Insofar as synaesthesia is relatively rare, there is interest in identifying methods by which it can be experimentally induced in the laboratory. The experimental induction of synaesthesia has the potential to shed light on the cognitive, neurophysiological, and neurochemical mechanisms that give rise to this condition (Cohen Kadosh, Henik, Catena, Walsh, and Fuentes, 2009; Luke, Terhune, and Friday, 2012). Induction methods also raise important questions regarding what can and should be considered synaesthesia and which demarcation criteria should be taken as paramount for discriminating synaesthesia from other conditions.

The present chapter reviews our current knowledge regarding the induction of synaesthesia in non-synaesthetes. First, we consider the criteria by which synaesthesia is currently defined with a view to using such criteria when we adjudicate whether different induced synaesthesias constitute genuine forms of this condition. We will describe three induction methods: training, 
Terhune, Luke, \& Cohen Kadosh, 2017, chapter in Sensory Blending: On synaesthesia and other phenomena

posthypnotic suggestion, and pharmacological agents. In each case, we adopt a critical stance whether the phenomenon induced by a particular method meets consensus criteria for synaesthesia and draw attention to its prospects and limitations. We conclude by contrasting the different methods and considering the implications of the experimental induction of synaesthesia for our understanding of the cognitive and neural mechanisms of this condition.

\subsection{Criteria and characteristics of synaesthesia}

When considering induced synaesthesias, it is crucial to evaluate their veracity; that is, whether they represent the same or similar phenomenon as congenital synaesthesia. To do so, we need a set of criteria by which synaesthesia can be identified. In this section we describe first the consensus criteria by which synaesthesia can be demarcated from other phenomena. We next consider what synaesthesia characteristics should be expected to be present in induced synaesthesias. For the sake of convenience, we will throughout refer to different induced synaesthesias as synaesthesias, rather than qualifying this term (e.g., alleged synaesthesias) each time we use it. Nevertheless, we reserve judgement as to whether these phenomena meet accepted criteria for this condition.

\subsubsection{Criteria}

There is still debate about the criteria by which synaesthesia can be discriminated from other experiences or associations, such as crossmodal correspondences. For example, human nonsynaesthetes, as well as chimpanzees (Ludwig, Adachi, and Matsuzawa, 2011), display pitchluminance correspondences, with higher-pitch tones being associated with higher luminance; this 
Terhune, Luke, \& Cohen Kadosh, 2017, chapter in Sensory Blending: On synaesthesia and other phenomena

phenomenon is highly prevalent in human non-synaesthetes and is not an accepted form of synaesthesia (Deroy and Spence, 2013). Nevertheless, there is an emerging set of criteria that are widely endorsed by the majority of synaesthesia researchers. Synaesthesia is often argued to be characterized by at least four criteria: 1) an atypical, ancillary conscious experience (e.g., colour) in response to a stimulus (e.g., a numeral); 2) a high degree of consistency in the inducerconcurrent associations; 3 ) a high degree of involuntariness (or automaticity) in the coupling of the inducer and concurrent and by which the concurrent breaches conscious awareness and impacts cognition; and 4) a high degree of specificity of the inducer and the concurrent (Deroy and Spence, 2013; Ward, 2013; Ward and Mattingley, 2006; see also Colizoli, Murre, and Rouw, 2014, for the application of demarcation criteria to trained synaesthesia).

A neglected question is whether induced synaesthesias should reasonably be expected to meet all of the foregoing criteria. That is, would we accept phenomena that meet certain criteria, but not others, as synaesthesia? For instance, if a synaesthete is not reliable over time in her inducer-concurrent associations but these associations are still automatic and accessible to consciousness, and perhaps even specific (at a particular time point), would we accept this as synaesthesia? This question is beyond the scope of the present chapter, but warrants attention (Simner, 2012). At present, it suffices to say that if one criterion is not met, we should not be too quick to discount a particular form of synaesthesia. Furthermore, certain criteria (conscious accessibility and automaticity) may be more fundamental than others (consistency and specificity).

Related to this question is the issue as to which criteria and characteristics should be expected in induced synaesthesias. This question has two strands: first, to what extent can the consensus criteria reviewed above be meaningfully applied to induced synaesthesias?; second, 
Terhune, Luke, \& Cohen Kadosh, 2017, chapter in Sensory Blending: On synaesthesia and other phenomena

independent of these criteria, should induced synaesthesias be expected to also elicit other characteristics found in synaesthetes? With regard to the first question, it is useful to distinguish between characteristics of synaesthesia that originate with the initial experience of synaesthesia (synaesthesia-specific) and those that are produced by the consolidation process wherein synaesthetic associations are repeatedly associated and strengthened over time (consolidationspecific). This distinction has not been empirically addressed to the best of our knowledge, thus at present it is unclear which characteristics and criteria can discriminate synaesthesia and crossmodal correspondences at early (pre-consolidation) and later (post-consolidation) stages of synaesthesia.

Consider, for instance, the criterion of consistency. At an early developmental stage, when a congenital synaesthete first begins to experience synaesthesia, we might expect that her inducerconcurrent associations would not be as consistent as the corresponding associations of adult synaesthetes. In other words, it is plausible that consistency emerges through a consolidation process over time. Data by Simner and colleagues (Simner, Harrold, Creed, Monro, and Foulkes, 2009), which shows that synaesthete children become more consistent in their grapheme-colour associations over time, is consistent with this speculation. Thus, early-stage synaesthesia may not be expected to be as consistent as late-stage synaesthesia. This argument similarly applies also to the criterion of specificity, as inducer-concurrent pairings may become more specific or specialized as part of a consolidation process. On the other hand, conscious access and automaticity are not necessarily specific to late-stage synaesthesia although this has not yet been systematically studied to our knowledge.

Induced synaesthesias can be easily likened to early-stage synaesthesia. Accordingly, it might be that we shouldn't necessarily expect to observe consistency and specificity in induced 
Terhune, Luke, \& Cohen Kadosh, 2017, chapter in Sensory Blending: On synaesthesia and other phenomena

synaesthesias, particularly in those where the inducer-concurrent pairings manifest on their own.

When we contrast induced and congenital synaesthesias below, we take account of the four aforementioned criteria, as well as other characteristics of synaesthesia, but it is important to bear in mind that induced synaesthesias may more closely approximate early-stage synaesthesias and thus may not meet all of the criteria of late-stage synaesthesias.

\subsubsection{Characteristics}

Aside from the demarcation criteria of synaesthesia, it is important to consider whether induced synaesthesias should exhibit other characteristics observed in synaesthetes. Before doing so, we would like to draw a distinction between characteristics that are specific to the online experience of synaesthesia (e.g., grapheme-colour consistency) and those that are not, such as enhanced visual processing (Banissy, Walsh, and Ward, 2009; Banissy, Tester, Muggleton, Janik, Davenport, Franklin, Walsh, and Ward, 2013; Barnett et al., 2008; Terhune, Song, Duta, and Cohen Kadosh, 2014; Yaro and Ward, 2007) and memory (Rothen et al., 2012). Consistency and other demarcation criteria are specific to the online experience of synaesthesia, whereas the latter are largely independent of these experiences and seem to represent markers of broader differences in visual cortex associated with the synaesthesia phenotype (Rothen et al., 2012; see also Terhune, Rothen, and Cohen Kadosh, 2013). Accordingly, we should not expect to observe non-specific characteristics in induced synaesthesias.

There are certain characteristics of synaesthesia that are not used as demarcation criteria for this condition, but which may still be expected in induced synaesthesias if they are genuine. For instance, it has been shown that synaesthetes typically display implicit bidirectionality, wherein concurrent stimuli trigger implicit inducer representations (e.g., colours triggering numbers) 
(Cohen Kadosh and Henik, 2007). This effect may occur as a result of the consolidation process by which graphemes and colours get repeatedly bound and thus may not be present in early or induced synaesthesias. Nevertheless, it is worth investigating and, if observed, will surely strengthen the case for the veracity of induced synaesthesias. An additional characteristic of synaesthesia is that of colour opponency in synaesthetic photisms (Nikolie, Lichti, and Singer, 2007). Hue-selective neurons in different regions of visual cortex have colour-opponent receptive fields such that cells that are excited by a particular colour (e.g., red) are inhibited by its opponent colour (e.g., green) (e.g., Zeki, 1980). Nikolić and colleagues found that synaesthetes were slower to name the colour of incongruently coloured graphemes when the incongruent colour was in an opponent colour as opposed to when it was in a non-opponent colour. They argued that the results suggest the involvement of early visual processing, perhaps involving V1, and point to the perceptual basis of synaesthesia. If induced synaesthesia is genuine, we might expect it to relate to colour-opponency effects. Finally, synaesthetes have also been shown to vary in the perceived visuospatial location of colour photisms, with some synaesthetes experiencing colours as endogenous images or representations (associators) and others as vivid percepts that are spatially colocalized with the inducer (projectors) (Dixon, Smilek, and Merikle, 2004; Ward, Li, Salih, and Sagiv, 2007), with evidence for neurophysiological differences (Cohen, Weidacker, Tankink, Scholte, and Rouw, 2015; Rouw and Scholte, 2010; Terhune, Murray, Near, Stagg, Cowey, and Cohen Kadosh, 2015; van Leeuwen, den Ouden, and Hagoort, 2011). Although we might not expect such effects, observing individual differences among induced synaesthetes increases the likelihood that genuine synaesthesia has been produced. These represent a few examples of characteristics of synaesthesia that may or may not be expected to be present in induced synaesthesias. 


\subsection{Induced synaesthesias}

Induced synaesthesias refer to instances in which synaesthesia or a synaesthesia-like phenomenon is temporarily elicited through exposure to an agent or manipulation. It is instructive to contrast these with acquired synaesthesias, which include those that develop in adulthood in the wake of some type of event (e.g., head injury) and which are experienced for an extended period of time. For instance, it has been shown that synaesthesias can be acquired through stroke (Fornazzari, Fischer, Ringer, and Schweizer, 2012; Ro et al., 2007) or perhaps sensory substitution (Ward and Wright, 2012) and have been reported to spontaneously occur during migraine (Alstadhaug and Benjaminsen, 2010). Below we describe three forms of induced synaesthesias: those that occur as a result of training, posthypnotic suggestion, and those that occur following the ingestion of pharmacological agents.

\subsubsection{Training}

A number of studies have explored the possibility of producing synaesthetic associations in nonsynaesthetes through training and whether these associations qualify as genuine synaesthesia (for a review, see Rothen and Meier, 2014). These studies involve repeatedly pairing two sets of stimuli that are typically associated in a form of synaesthesia (e.g., graphemes and colours) and examining whether training leads to behavioural or physiological responses to the stimuli in a manner typical of synaesthesia. In addition to exploring the possible induction of synaesthesia, researchers have been motivated to use this approach to include trained participants as a control group for synaesthetes (Cohen Kadosh et al., 2005; Elias, Saucier, Hardie, and Sarty, 2003; Nunn et al., 2002) to examine whether synaesthetic effects are just a product of semantic associations 
Terhune, Luke, \& Cohen Kadosh, 2017, chapter in Sensory Blending: On synaesthesia and other phenomena

or memory. Moreover, in a number of documented cases, synaesthetes' specific grapheme-colour pairs appear to have been determined by exposure to coloured graphemes in early childhood (Hancock, 2006; Witthoft and Winawer, 2006, 2013). Accordingly, investigating whether synaesthesia can be induced through training also has a direct bearing on the learning mechanisms at play in developmental synaesthesia.

Multiple studies have shown that grapheme-colour association training can reproduce the behavioural markers of grapheme-colour synaesthesia. In a nice early example of this, Elias and colleagues (2003) compared a single grapheme-colour synaesthete with a single semantic-control and untrained controls. This study is unique insofar as the semantic-control had spontaneously developed grapheme-colour associations through exposure over an eight-year period to a crossstitching system in which a particular number signifies a particular thread colour. Strictly speaking, the development of the grapheme-colour associations in this case is spontaneous and not the product of an experimental manipulation as is the case with the training studies described below.

In this study, Elias and colleagues administered a series of behavioural tasks previously shown to discriminate controls and synaesthetes as well as multiple behavioural tasks during functional magnetic resonance imaging. The three tasks included a coloured grapheme Stroop task, a mathematical Stroop task, in which mathematical equations are followed by colour patches that are either congruent or incongruent with the correct answer (Dixon, Smilek, Cudahy, and Merikle, 2000), and a conscious priming task in which congruent or incongruent graphemes preceded colour patches in a colour-naming task (Mattingley, Rich, Yelland, and Bradshaw, 2001). Crucially, in all three tasks, the synaesthete and the semantic-control exhibited congruency effects characterized by slower responses on incongruent than congruent trials, 
Terhune, Luke, \& Cohen Kadosh, 2017, chapter in Sensory Blending: On synaesthesia and other phenomena

whereas none of the untrained controls displayed such effects. Indeed, the semantic-control displayed comparably sized, or numerically larger, congruency effects relative to the synaesthete in all conditions. In contrast, in the fMRI paradigms, the synaesthete displayed activation in left parietal and extrastriate visual cortex during auditory and visual numerical processing, respectively, whereas no such effects were observed in the semantic-control. Although caution is required when interpreting single-case results, these findings suggest that trained semantic associations between graphemes and colours are not producing the same cortical activation patterns observed in synaesthesia, or the conscious, involuntary experience of colour photisms that is a hallmark phenomenological property of synaesthesia.

A subsequent study that included trained non-synaesthetes similarly shows that they do not exhibit behavioural effects observed in congenital synaesthesia (Cohen Kadosh et al., 2005). In this study, non-synaesthetes were trained over five sessions to associate specific digits and colours and evidenced clear learning effects. The authors sought to investigate whether colours implicitly activate numerical magnitude representations in synaesthetes and the trained group. Participants completed a magnitude comparison task in which they had to judge which of two numbers was numerically larger. The numbers were presented in their associated synaesthetic colours (matched-colour condition) or in colours that were associated with a larger numerical distance (large-colour condition). Crucially, grapheme-colour synaesthetes were faster in the large colour than in the matched-colour condition, indicating that colour implicitly triggers a numerical representation, even though synaesthetes are not consciously aware of this implicit association. Of special interest in the present context is that the trained control group did not display this effect, although they did display a normal congruency effect (faster responses for the matched-colour condition). This indicates that whilst non-synaesthetes can be trained to associate 
Terhune, Luke, \& Cohen Kadosh, 2017, chapter in Sensory Blending: On synaesthesia and other phenomena

graphemes and colours, they not do not exhibit implicit bidirectionality, as is observed in congenital synaesthetes.

Meier and Rothen (2009) adopted a similar approach and investigated whether seven days of training would elicit the physiological concomitants of synaesthesia. After training, the trained controls completed a synaesthesia Stroop task and a synaesthesia conditioning task (Meier and Rothen, 2007). In the latter, a particular colour was paired with a startling sound, thereby producing a conditioned startle response to the colour, as measured by skin conductance response. The training produced a small, but significant, Stroop effect $(23 \mathrm{~ms})$; in contrast, unlike congenital synaesthetes (Meier and Rothen, 2007), the participants displayed a conditioning effect for colours, but not graphemes. One explanation for why this divergence arises is that the trained controls do not develop implicit bidirectional associations between colours and graphemes; that is, in synaesthetes, it may be that colour implicitly activates grapheme representations, which are then associated with the conditioned stimulus, whereas colour does not activate grapheme representations in trained controls (Cohen Kadosh et al., 2005).

A subsequent study by Rothen and colleagues (Rothen, Wantz, and Meier, 2011) sought to expand upon their previous study by contrasting two different types of training. In addition, the authors administered digit-colour and colour-digit priming tasks (Gebuis, Nijboer, and Van der Smagt, 2009) so as to examine bidirectionality effects in the wake of training as a follow-up to the studies described above. In a non-adaptive training schedule, participants judged whether digits were correctly or incorrectly coloured on the basis of predefined digit-colour associations. In contrast, the adaptive training schedule involved participants identifying the colour that was associated with an achromatic digit, according to the predefined associations. The digit was represented in the correct associated colour hue with altered luminance and participants had to 
judge whether the stimulus colour was brighter or darker than the colour associated with the digit. Feedback was given after both the initial digit as well as the coloured digit in the adaptive training schedule but not in the non-adaptive training schedule.

The authors found that participants displayed larger priming effects across both tasks after ten days of training. In addition, participants exhibited larger digit-colour than colour-digit priming effects, as might be expected given the results of Cohen Kadosh et al. (2005). Exploratory analyses further revealed that both groups displayed significant digit-colour priming effects post-training, whereas only the adaptive training group displayed a colour-digit priming effect. Importantly, as in other studies, none of the trained participants reported colour photisms in response to digits. Insofar as the authors found no differences across tasks as a function of the type of training, caution should be exerted in interpreting these results. Nevertheless, these findings suggest that the adaptive training schedule may give rise to bidirectionality effects observed in synaesthetes (Gebuis et al., 2009). At the same time, it should be noted that the largest digit-colour priming effect (observed in the adaptive training group; $\sim 37 \mathrm{~ms}$ ), and the largest colour-digit priming effect (observed in the non-adaptive training group; $\sim 18 \mathrm{~ms}$ ) stand in stark contrast to the far larger priming effects observed in synaesthetes (digit-colour priming: 135 ms; colour-digit priming: $\sim 134 \mathrm{~ms}$; Gebuis et al., 2009). Given the results, it is unclear whether the adaptive training produces stronger effects and, because multiple components of the training were different across the two methods, it is difficult to determine which component(s) of training are crucial for any benefit with this method. Nevertheless, this study does suggest that adaptive training may be used to replicate behavioural markers of synaesthesia.

The remaining studies that we will describe adopt a more naturalistic approach by having participants complete tasks that should produce synaesthesia-like associations rather than 
Terhune, Luke, \& Cohen Kadosh, 2017, chapter in Sensory Blending: On synaesthesia and other phenomena

explicitly attempting to train such associations. Colizoli and colleagues had participants read one or more books in which four letters were uniquely coloured (Colizoli, Murre, and Rouw, 2012). Participants subsequently completed multiple behavioural tasks that are potentially diagnostic of synaesthesia including a synaesthesia Stroop task, a perceptual crowding task, and a surprise letter-colour pair recollection test. The crowding task involved viewing a group of letters with one unique target letter embedded within the display and identifying the target letter; a control task involved untrained letters. The surprise test involved querying participants about the grapheme-colour associations four to six months after the conclusion of the study.

In line with some of the foregoing studies, Colizoli et al. (2012) observed that trained participants displayed a larger Stroop effect after training. A subset of participants who read multiple books displayed larger Stroop effects as more books were read, but the magnitude of this change was unrelated to the number of words read, nor was the number of words read related to the overall Stroop effect. Accordingly, this study provides mixed results regarding whether the amount of training is a determining factor of the magnitude of the post-training Stroop effect. One related notable finding is that there were marked individual differences in the magnitude of the Stroop effect after training with Stroop effects ranging from -26 to $185 \mathrm{~ms}$ : this points to considerable variability in the extent to which training elicits Stroop effects and suggests that certain individuals are more prone to these effects. Finally, the extent to which participants reported experiencing colours when seeing letters was unrelated to Stroop effects.

The results of the crowding and recall tests were less conclusive. Participants were unable to recall all of the letter-colour pairs in the recall test, but recalled with high accuracy the colours paired with each number. No controls were included in this task and thus it is unclear how remarkable this result is. Similarly, trained participants did not outperform controls on the 
crowding task, unlike synaesthetes (Ward, Jonas, Dienes, and Seth, 2010). Moreover, there is disagreement regarding the interpretation of superior performance among synaesthetes in this task (Ward et al., 2010), and so it is unclear whether training here is failing to reproduce a perceptual or attentional benefit conferred by synaesthesia. This study, as above, confirms that training can produce Stroop effects, but not other features of synaesthesia, and is equivocal regarding the extent to which training strengthens grapheme-colour associations.

Another study (Kusnir and Thut, 2012) shows that training can produce further behavioural effects that parallel those observed in synaesthetes. In this study, participants completed a lettersearch task, in which they searched for target letters among an array and judged whether the target was to the left or the right of a central fixation cross. Certain target letters were more often associated with specific colours to facilitate statistical semantic learning over time; half of the participants were informed of the bias in the stimulus presentation. Participants' search times were faster for biased (congruent and incongruent) than unbiased stimuli, suggesting strong grapheme-colour binding. However, although the search time was faster over time, it did not vary over time for the different types of stimuli. In other words, the grapheme-colour binding effect appears to occur relatively early, although it is difficult to say when it became robust.

Stroop interference was associated with grapheme-colour binding strength in the aware group, but participants did not display an overall Stroop effect as a result of training, nor did the best learners exhibit the largest Stroop effect. An interesting result is that participants displayed larger interference in the search task when target colours were in opponent colours relative to the grapheme-colour association as opposed to non-opponent colours. For example, if 6 was paired with the colour red, participants were slower in responding when 6 was printed in green ink. This result is notable, because it is also displayed by synaesthetes (Nikolić et al., 2007) and 
Terhune, Luke, \& Cohen Kadosh, 2017, chapter in Sensory Blending: On synaesthesia and other phenomena

because it implicates early visual processing, thereby suggesting a perceptual component to the processing of grapheme-colour associations (and potentially indirectly corroborating some of Colizoli et al.'s [2016]).

To summarize, as is the case with the other studies, this study produced somewhat equivocal results. Training did not elicit Stroop effects, but did elicit robust grapheme-colour binding and colour-opponency effects. The authors argue that explicit instructions such as those in Meier and Rothen (2009) may be more likely to produce associations at a more conceptual level, and thus larger Stroop effects, whereas implicit training may be more likely to elicit perceptual associations. However, this is at odds with the interpretation of synaesthetic Stroop effects by Nikoliç and colleagues (2007), who argue that synaesthetic Stroop effects reflect a perceptual component, as exemplified by colour-opponency effects, as well as a smaller, semantic component. Further research is clearly needed to test these varying interpretations and to assess the extent to which the grapheme-colour associations induced in Kusnir and Thut's (2012) study resemble genuine synaesthesia.

It is plausible that behavioural tasks are insufficiently powerful in detecting induced synaesthesia and that neuroimaging methods may be more sensitive in capturing traininginduced synaesthesia. Toward this end, Colizoli and colleagues recently investigated the neurophysiological effects of their coloured-letter training paradigm using fMRI (Colizoli, Murre, Scholte, van Es, Knapen, and Rouw, 2016). The authors found that training with coloured books over approximately 20 days did not produce grapheme-colour consistency at the level typically observed in developmental synaesthetes. Similarly, none of the participants subjectively reported experiencing colour photisms. As in some previous studies, training was associated with a significantly greater Stroop effect post-training relative to baseline; moreover, as commonly 
Terhune, Luke, \& Cohen Kadosh, 2017, chapter in Sensory Blending: On synaesthesia and other phenomena

observed in this literature, the magnitude of post-training Stroop interference ( $\sim 26 \mathrm{~ms})$ was substantially lower than that observed in congenital synaesthetes and was unrelated to imagery and subjective colour experiences.

Functional correlates of these effects are difficult to interpret (Colizoli et al., 2016). The authors found that activation of $\mathrm{V} 4$, a region widely implicated in colour processing and synaesthesia (Ward, 2013), was greater for coloured (untrained) letters than trained and untrained (achromatic) letters, as would be expected. However, surprisingly, trained letters were characterized by less V4 activation than untrained letters and trained letters were also associated with a negative BOLD response relative to untrained letters in primary visual cortex. Moreover, visual cortex activation patterns were unrelated to Stroop effects, although there was some indication that the tendency to have associator experiences in response to graphemes was associated with greater V4 differences between trained and untrained letters. By implicating primary visual cortex in training of synaesthesia, these results are encouraging but preliminary. In particular, the result suggest that training grapheme-colour synaesthesia might elicit changes in the brain's response to graphemes but the meaning of these effects is somewhat equivocal and it remains unclear how they may relate to, or inform our understanding of, developmental synaesthesia.

A final recent study suggests that the limited results of previous studies may be driven by insufficient training (Bor, Rothen, Schwartzman, Clayton, and Seth, 2014). This study aimed to couple greater ecological validity with a rigorous and diverse training schedule that changed over time. Fourteen participants underwent 9 weeks of training involving a range of cognitive tasks in which specific grapheme-colour pairs were repeatedly presented; they were also given "homework" consisting of books with coloured letters (Colizoli et al., 2012) and performance 
Terhune, Luke, \& Cohen Kadosh, 2017, chapter in Sensory Blending: On synaesthesia and other phenomena

gains were reinforced with monetary compensation. After training, over $50 \%$ of participants reported phenomenological associations resembling synaesthesia but only 1 participant reported projector-like perceptual experiences (these effects did not seem to be related to individual differences in imagery at baseline). Interestingly, nearly all participants spontaneously developed ordinal linguistic personification, in which certain characteristics (aggression) are tied to specific graphemes; these phenomena seem to closely resemble similar effects in congenital synaesthetes (Simner and Holenstein, 2007). Participants also displayed greater Stroop colour-naming effects post-training relative to baseline although this effect seemed to be specific to semantic associations. Critically, for the first time, to our knowledge, the authors also reported that participants displayed superior grapheme-colour consistency post-training than at baseline and at levels typically observed in developmental synaesthetes (Rothen et al., 2013), as well as other synaesthesia-specific effects (Bor et al., 2014).

The training studies undertaken to date clearly demonstrate that grapheme-colour associations can be induced in non-synaesthetes through training (Rothen and Meier, 2014). However, trained participants do not reliably display behavioural markers of synaesthesia, such as greater Stroop effects post-training (Kusnir and Thut, 2012; Rothen et al., 2011), implicit bidirectionality (Cohen Kadosh et al., 2005), or perceptual crowding (Colizoli et al., 2012). Moreover, when effects are observed, they do not display the same behavioural response patterns (Rothen et al., 2011), and/or they are smaller in magnitude than those observed in genuine synaesthetes (Dixon et al., 2004; Gebuis et al., 2009). For example, the largest Stroop effect in trained participants to the best of our knowledge was $\sim 57 \mathrm{~ms}$ (Colizoli et al., 2012), which is comparable to that observed in congenital associator synaesthetes (Dixon et al., 2004; Ward et al., 2007). In contrast, when both associator and projector synaesthetes are included, the 
Terhune, Luke, \& Cohen Kadosh, 2017, chapter in Sensory Blending: On synaesthesia and other phenomena

magnitude of the Stroop effect has been found to vary from $\sim 90 \mathrm{~ms}$ (Dixon et al., 2004) to $\sim 130$ ms (Ward et al., 2007). Inconsistent or limited findings may stem from differences in training regimens across studies, with comprehensive training regimens seeming to produce the most compelling results (Bor et al., 2014). Moreover, only one study (Bor et al., 2014) has observed reports of conscious experiences of colour associations and even this was rare and transient. Three studies have shown that trained participants do not display the same patterns of neural (Elias et al., 2003; Nunn et al., 2002) or physiological (Meier and Rothen, 2009) effects as genuine synaesthetes, although preliminary research has implicated changes in primary visual cortex in trained synaesthesia (Colizoli et al., 2016). Whilst we do not want to dismiss the possibility that future training studies will successfully induce synaesthesia, we think it is prudent to interpret the present results as providing only tentative evidence for inducing certain features of synaesthesia (see also Rothen and Meier, 2014).

It is instructive to consider the trained associations relative to the demarcation criteria and characteristics of synaesthesia that we outlined above. First, there is evidence that training can produce consistency of grapheme-colour associations that rivals that observed in developmental synaesthetest (Bor et al., 2014) (other results are suggestive [Colizoli et al., 2012]). However, it could be argued that consistency is only a valuable measure when a sizeable number of grapheme-colour associations are trained because a small number of associations, as used in the training studies reviewed here, will be relatively easy to remember. Some studies to date have used canonical colours (Bor et al., 2014), which may further facilitate recall. Nevertheless, the criterion of consistency appears to have been met at least in a preliminary fashion. Similarly, we believe the criterion of automaticity has been met by training studies, but only to an extent. A number of studies have highlighted the fact that Stroop effects in trained groups are comparable 
to those in congenital synaesthetes (e.g., Elias et al., 2003) and/or that Stroop-type effects should not be used as diagnostic indicators of synaesthesia because they are unable to distinguish between semantic associations and those produced by involuntary colour photisms (Colizoli et al., 2012). We agree with this in part but it is important to recall that Stroop effects in trained groups of a comparable magnitude to those observed in synaesthetes do not always occur and no training study has observed Stroop effects comparable to those seen in projector synaesthetes (Dixon et al., 2004; Ward et al., 2007). One study has provided evidence that the criterion of conscious accessibility has been met as the majority of participants reported conscious experiences of colour photisms; however, insofar as only one participant reported projector-like experiences, there is not yet clear evidence for the individual differences typically observed in congenital synaesthetes. We revisit whether these results warrant revision of demarcation criteria later in this chapter.

When comparing congenital and induced synaesthetes, it is important to consider the confound of differential consolidation in this line of research. It is entirely possible that trained synaesthetes would display each of the behavioural, phenomenological, and neural markers of congenital synaesthesia if their associations were continuously reinforced (and consolidated) with long-term experience. Given the length of time that most synaesthetes have experienced this condition, this appears to be an insurmountable confound. The trained participant in Elias and colleagues' (2003) study exhibited grapheme-colour associations and underwent a form of training over eight years and still did not display the neural markers of synaesthesia, suggesting that long-term consolidation may not actually be sufficient to induce genuine synaesthesia. This was a case study and thus this result is far from conclusive. However, this confound should be considered when evaluating the authenticity of trained and other induced synaesthesias. 
One approach to advancing research in this domain may be to couple training regimens with non-invasive brain stimulation techniques, which have been shown to enhance the impact of cognitive training (Krause \& Cohen Kadosh, 2013). For example, we and others have consistently shown that concurrent application of transcranial electrical stimulation can be used to enhance the effects of training on a range of cognitive and perceptual functions, with longlasting effects in specific cases (Fertonani, Pirulli, and Miniussi, 2011; Snowball, Tachtsidis, Popescu, Thompson, Delazer, Zamarian, Zhu, and Cohen Kadosh, 2013; Cappelletti, Gessaroli, Hithersay, Mitolo, Didino, Kanai, Cohen Kadosh, and Walsh, 2013; Looi, Duta, Brem, Huber, Nuerk, \& Cohen Kadosh, 2016; Reis, Schambra, Cohen, Buch, Fritsch, Zarahn, Celnik, \& Krakauer, 2009). Elsewhere, we have shown that synaesthetes display elevated cortical excitability in primary visual cortex (Terhune, Tai, Cowey, Popescu, and Cohen Kadosh, 2011; Terhune, Murray, Near, Stagg, Cowey, and Cohen Kadosh, 2015). Thus, specifically coupling non-invasive brain stimulation methods for enhancing excitability in primary visual cortex with rigorous cognitive training may provide an approach that is both methodologically robust but also grounded in contemporary knowledge of the neurophysiology of congenital synaesthesia.

\subsubsection{Posthypnotic suggestion}

A second method for the induction of synaesthesia involves the use of posthypnotic suggestion with highly suggestible individuals. Here we introduce a few of the hallmark features of hypnosis and posthypnotic suggestion and then review the studies that have used this approach to induce synaesthesia.

Hypnosis consists of a set of procedures including an induction and one or more suggestions (Oakley and Halligan, 2009; Terhune and Cohen Kadosh, 2012a). Inductions vary but generally 
Terhune, Luke, \& Cohen Kadosh, 2017, chapter in Sensory Blending: On synaesthesia and other phenomena

involve instructions and suggestions to promote minimized awareness of one's environment, reduced metacognition, and perceived effortless attention towards the instructions of the experimenter (Brown, Antonova, Langley, and Oakley, 2001). Suggestions are verbal statements administered by an experimenter for specific changes in affective, cognitive, motor, or perceptual functions. They are typically conveyed in such a manner that they invite passive responses that are experienced as happening to an individual, rather than actions or representations that an individual willingly performs or produces, so as to augment the extra-volitional phenomenology of hypnotic responding (Bowers, 1981; Spanos and Gorassini, 1984). An example of a hypnotic suggestion for a visual hallucination may be, "When you open your eyes in a few moments, you will look at the computer monitor in front of you and see a red circle." Posthypnotic suggestions are suggestions for alterations in a particular function following a hypnotic de-induction. Although not originally intended for this purpose, most hypnosis researchers favour using such suggestions instead of regular hypnotic suggestions to dissociate the effects of the suggestion from those of the hypnotic induction. Specifically, it has been repeatedly shown that a hypnotic induction impairs attention in highly suggestible individuals (Egner and Raz, 2007), or at least a subset of highly suggestible individuals (Marcusson-Clavertz, Terhune, and Cardeñ̃a, 2012; Terhune, Cardeña,a, and Lindgren, 2011b). Posthypnotic suggestions circumvent the possible confound of impaired attention that may impact responding to hypnotic suggestions.

The most well established fact about hypnosis is that people display marked variability in their responsiveness to hypnotic suggestions (Hilgard, 1965). Hypnotic suggestibility varies widely in the general population with approximately $10-15$ per cent of individuals displaying low hypnotic suggestibility, 10-15 per cent displaying high hypnotic suggestibility, and the remaining 70-80 per cent of the population exhibiting a moderate level of suggestibility 
(Laurence, Beaulieu-Prếvost, and du Chếnế, 2008). Hypnotic suggestibility is typically measured with one or more behavioural scales that comprise a series of hypnotic suggestions (for a review, see Woody and Barnier, 2008). These instruments are necessary for reliably screening participants and identifying individuals of different levels of hypnotic suggestibility, who cannot be differentiated by questionnaires or self-report. Most hypnosis research involves the use of highly suggestible individuals, including low-suggestible and/or medium-suggestible participants as a control group.

The first study demonstrating the posthypnotic induction of synaesthesia was reported by Cohen Kadosh and colleagues (Cohen Kadosh et al., 2009). In this study, the authors administered a posthypnotic suggestion to highly suggestible participants to experience projector grapheme-colour synaesthesia for six grapheme-colour pairs. Control participants received instructions for the same digit-colour associations but with no posthypnotic suggestions. Under the cover of the posthypnotic suggestion and in a control condition, participants completed a digit detection task in which they had to judge whether a digit was presented on a monitor or not (Smilek, Dixon, Cudahy, and Merikle, 2001). Digits were presented in black ink against a coloured background, which was either congruent or incongruent with the colour associated with the digit. A previous study found that congenital synaesthetes were poorer at detecting whether a digit was present during the congruent condition (Smilek et al., 2001). The induced synaesthetes, but not the control groups, displayed this same response pattern. In addition, whilst under the posthypnotic suggestion, they also reported experiencing colour photisms in response to graphemes outside of the laboratory. This study nicely demonstrates that both the behavioural and experiential markers of synaesthesia can be induced in the laboratory using posthypnotic suggestion. 
Terhune, Luke, \& Cohen Kadosh, 2017, chapter in Sensory Blending: On synaesthesia and other phenomena

Recently, a second study failed to observe behavioural markers of synaesthesia in nonsynaesthetes given a posthypnotic suggestion for synaesthesia (Anderson, Seth, Dienes, and Ward, 2014). Null results are challenging to interpret and thus we can only speculate as to the reasons for their failure to observe induced synaesthesia. First, their procedure for screening participants was not as rigorous as most hypnosis studies, including that by Cohen Kadosh et al. (2009), which typically include two rounds of hypnotic suggestibility measurement to ensure that participants are sufficiently highly suggestible (Woody and Barnier, 2008). In addition, the participants were not screened prior to the experiment for their ability to respond to hallucination or synaesthesia suggestions. Highly suggestible individuals display pronounced variability in hypnotic responding and some are incapable of responding to hallucination suggestions (Szechtman, Woody, Bowers, and Nahmias, 1998). Combined, these two features already strongly suggest that only a small subset of the participant pool in this study would be responsive to such a suggestion. Finally, the authors used an embedded figures task, in which synaesthetic concurrent colours are presumed to facilitate "pop-out" of embedded higher-order stimuli in an array of stimuli. This task is problematic as synaesthetes have not been reliably shown to outperform controls in this task (for a review, see Ward et al., 2010). It is imperative that research attempting to assess the veracity of induced synaesthesia needs to use well-established tasks that reliably distinguish synaesthetes from controls.

In another study, we expanded upon the approach of Cohen Kadosh et al. (2009) and used posthypnotic suggestion to induce different phenomenological subtypes of synaesthesia (Terhune and Cohen Kadosh, 2012b). We were motivated to conduct this experiment because it remains unclear whether the observed behavioural markers of associator and projector synaesthesia (Dixon et al., 2004) are reliable (Hupé, Bordier, and Dojat, 2011; Ward et al., 2007) and are 
Terhune, Luke, \& Cohen Kadosh, 2017, chapter in Sensory Blending: On synaesthesia and other phenomena

actually the product of individual differences in the perceived visuospatial location of colour photisms. We first doubly-screened participants (Woody and Barnier, 2008) and then identified highly suggestible individuals who were subjectively responsive to hypnotic suggestions for either associator or projector grapheme-colour synaesthesia. Next, we administered posthypnotic suggestions to associate four numbers with four colours and to experience colours as either spatially colocalized with graphemes (projectors) or as mental images (associators) to the respective two subgroups who were responsive to these types of suggestions. Participants completed two Stroop colour-naming tasks previously shown to discriminate associators and projectors (Dixon et al., 2004): in the stimulus colour-naming task, they named the colour of the digit, whereas in the photism colour-naming task, they named the colour associated with the digit.

The behavioural and phenomenological responses of the induced synaesthetes in this study closely resemble those of the congenital synaesthetes. In both groups, projector synaesthetes displayed larger congruency effects in the stimulus colour-naming task than associator synaesthetes (Dixon et al., 2004), whereas the two groups did not differ in the photism colournaming task. Attempting to identify the stimulus colour is presumably more difficult for projectors because their colour photisms are perceived to be spatially colocalized with the stimulus and thereby elicit greater response conflict when naming the stimulus colour. Across conditions, induced synaesthetes displayed comparable performance, as measured by response latencies, to congenital synaesthetes. Indeed, induced projectors actually displayed numerically, but not significantly, larger Stroop effects in the stimulus colour-naming task than congenital projectors. Below we maintain that this unexpected result is actually consistent with the induction of genuine synaesthesia. The phenomenological reports nicely complement the 
Terhune, Luke, \& Cohen Kadosh, 2017, chapter in Sensory Blending: On synaesthesia and other phenomena

behavioural results. Congenital and induced synaesthetes reported similar subjective levels of both the involuntariness and the vividness of colour photisms during the Stroop tasks, but congenital synaesthetes actually reported experiencing colour photisms more often (some induced synaesthetes did report colour photisms on 100 per cent of the experimental trials, though). Two other effects are worth mentioning in the present context. Two highly suggestible participants reported spontaneous colour photisms for numbers that were not paired with a colour by posthypnotic suggestion and a single highly suggestible participant reported explicit bidirectionality such that colours triggered conscious experiences of the paired number. Insofar as these variables were not formally assessed in this experiment, the extent to which these experiences spontaneously occur is unclear; nevertheless, these effects highlight the range of synaesthetic effects that could potentially be induced using posthypnotic suggestion.

This study corroborates and extends the results of Cohen Kadosh and colleagues (2009). First, it replicates the previous result that posthypnotic suggestion can be used to induce synaesthesia-like behavioural and phenomenological response patterns. These studies indicate that the behavioural and phenomenological markers of synaesthesia can be reproduced using posthypnotic suggestion. Second, it extends the results of Cohen Kadosh and colleagues by showing that different phenomenological subtypes of synaesthesia can be induced. Finally, it demonstrates that variability in response patterns on Stroop tasks among synaesthetes is a product of individual differences in the perceived visuospatial location of colour photisms, and thereby provides valuable information regarding individual differences in grapheme-colour synaesthesia. Accordingly, these studies nicely complement other instrumental hypnosis research (Oakley and Halligan, 2009) and present a method by which synaesthesia can be experimentally 
Terhune, Luke, \& Cohen Kadosh, 2017, chapter in Sensory Blending: On synaesthesia and other phenomena

modulated in the laboratory. The use of such a method has considerable potential in tackling a wide range of questions regarding the characteristics and mechanisms of synaesthesia.

These studies raise the question whether posthypnotic suggestion is producing genuine synaesthesia. The available evidence suggests the affirmative. Posthypnotic suggestion is capable of reproducing the behavioural and phenomenological markers of congenital synaesthesia.

Posthypnotic synaesthetes also display behavioural effects of comparable magnitude, suggesting a similar degree of involuntariness, which is corroborated by the phenomenological reports. However, whether congenital and induced synaesthesia are occurring through similar or overlapping neural mechanisms remains an open question that needs to be addressed empirically. Hypnotic suggestions for colour hallucinations have previously been shown to produce greater activation in V4 (a region repeatedly implicated in the representation of synaesthetic colour photisms (Brang, Hubbard, Coulson, Huang, and Ramachandran, 2010; Hubbard, Arman, Ramachandran, and Boynton, 2005)) than colour imagery (Kosslyn, Thompson, CostantiniFerrando, Alpert, and Spiegel, 2000; McGeown et al., 2012). This indicates that colour experiences associated with V4 activation can be produced using posthypnotic suggestion. One notable finding that is especially compelling here is that induced projector synaesthetes displayed numerically larger Stroop effects in the stimulus colour-naming task than congenital projector synaesthetes, suggesting elevated involuntariness of colour photisms relative to congenital projector synaesthetes (this was also found by Cohen Kadosh et al. (2009)). This finding seems peculiar at first glance but we think it actually provides further evidence for the authenticity of posthypnotically induced synaesthesia. Congenital synaesthetes are repeatedly exposed to incongruent grapheme colours pairs in product labels, street signs, web content, and so on. In turn, it is necessary for them to develop strategies to manage this conflict in their daily lives, 
Terhune, Luke, \& Cohen Kadosh, 2017, chapter in Sensory Blending: On synaesthesia and other phenomena

which aids them when completing a synaesthesia Stroop task. In contrast, grapheme-colour associations are novel experiences for induced synaesthetes and this group will not have access to a repertoire of conflict management strategies to attenuate synaesthesia-specific response conflict, and in turn, Stroop effects. Indeed, many of our synaesthetes reported that the experience was so novel and unusual that the Stroop task was especially difficult. Accordingly, it may be that numerically greater Stroop effects in induced synaesthetes are a consequence of having only experienced grapheme-colour associations for a short duration.

The posthypnotic induction of synaesthesia has broader implications for the neural mechanisms underlying this condition. If posthypnotic suggestion is temporarily and instantaneously producing synaesthesia, these results are at odds with the position that synaesthesia is a direct result of greater anatomical connectivity between cortical structures supporting the processing of the inducer and the concurrent (cross-activation theory; Hubbard, 2007; Hubbard, Brang, and Ramachandran, 2011). It is highly unlikely that a posthypnotic suggestion is capable of producing excess structural brain connectivity within minutes, thus posthypnotic induction of synaesthesia suggests that theories postulating that anatomical connectivity plays a causal role in the development and online occurrence of synaesthesia are incorrect or at least incomplete (Cohen Kadosh et al., 2009).

If we momentarily accept that a posthypnotic suggestion is producing genuine synaesthesia, there are two plausible ways by which these results can be reconciled with cross-activation theory. One possibility is that there are two (or more) sets of mechanisms by which synaesthesia can be produced. On this account, synaesthesia can occur through both a genetic predisposition for enhanced connectivity, but also through a second mechanism, such as cortical disinhibition (Cohen Kadosh et al., 2009). According to this view, synaesthesia results from a disruption of 
Terhune, Luke, \& Cohen Kadosh, 2017, chapter in Sensory Blending: On synaesthesia and other phenomena

cortical inhibition, producing conscious awareness of visual information that is normally inhibited (Cohen Kadosh and Walsh, 2008; Eagleman and Goodale, 2009). A second possibility is that both congenital and induced synaesthesia occur through disinhibition and that excess connectivity is a byproduct of the repeated binding of inducer and concurrent representations; that is, it is a consequence, rather than cause, of synaesthesia (Cohen Kadosh and Walsh, 2008). Determining which possibility is more plausible is ultimately an empirical issue. Nevertheless, coupled with other results that are at odds with cross-activation theory (Nikolie, Jurgens, Rothen, Meier, and Mroczko, 2011), the two studies described above call into question the viability of the cross-activation theory as a comprehensive account of synaesthesia.

One critique of this line of research is that the mechanisms underlying hypnosis are poorly understood and thus it remains unclear how hypnotic suggestion is effecting the synaesthetic response (Hubbard, 2011). We think that this line of argument sidesteps the value of the use of posthypnotic suggestion for studying synaesthesia. Although a comprehensive account of the neurocognitive mechanisms underlying hypnosis has yet to be advanced, there is emerging evidence that a reduction in prefrontal cortical activity (Dienes and Hutton, 2013) or a decoupling of prefrontal cortex with anterior cingulate cortex (Egner, Jamieson, and Gruzelier, 2005) or parietal cortex (Terhune, Cardeñ̃a, and Lindgren, 2011a) in highly suggestible individuals facilitates hypnotic responding, in particular the experience that responses are occurring extra-volitionally. A number of studies have also suggested candidate regions for the top-down modulation of alterations in conscious awareness following particular suggestions including orbitofrontal cortex and precuneus (Cojan et al., 2009; Mendelsohn, Chalamish, Solomonovich, and Dudai, 2008). Such regions, or perhaps other regions in the frontal-parietal 
Terhune, Luke, \& Cohen Kadosh, 2017, chapter in Sensory Blending: On synaesthesia and other phenomena

network, most likely play a top-down role in producing activation in V4 and other regions involved in the synaesthetic experience (Cohen Kadosh et al., 2009).

\subsubsection{Pharmacological agents}

The context in which non-synaesthetes seem most often to spontaneously experience synaesthesia is arguably under the influence of pharmacological agents, such as recreational drugs. In turn, the administration of such agents provides a potentially valuable method for inducing synaesthesia in a controlled environment (Luke and Terhune, 2013). This method has a number of clear methodological advantages over training and posthypnotic suggestion methods. First, unlike training, pharmacological agents may be expected to elicit synaesthesia in a relatively short period of time. Second, unlike posthypnotic induction, this method is expected to be effective with a relatively large proportion of the population, perhaps as high as 60 per cent (Luke et al., 2012; Tart, 1975). Finally, insofar as much is known about the neurotransmitter systems affected by pharmacological agents (Carhart-Harris et al., 2012), their use for inducing synaesthesia may lend insights into the neurotransmitters implicated in the induction, modulation, and disruption of synaesthesia, as well as the neuro-developmental origins of congenital synaesthesia.

In some cases, the neurotransmitter systems targeted by particular drugs are well known, as with the classical tryptamine psychedelics such as psilocybin, which are selective $5 \mathrm{HT}_{2 \mathrm{~A}}$ partial agonists (Lee and Roth, 2012). The neural mechanisms involved continue to be debated, but a recent fMRI study (Carhart-Harris et al., 2012) shows that, contrary to expectations, psilocybin decreases cerebral blood flow to key regions, specifically the thalamus, anterior and posterior cingulate cortex (ACC \& PCC), and medial prefrontal cortex (mPFC), the latter two of which are 
primary regions in the default mode network (Raichle et al., 2001). Significantly, the usual positive coupling between the $\mathrm{mPFC}$ and the PCC is also reduced during the intake of psilocybin. At the same time, some chemicals, known as promiscuous drugs, modulate a variety of neurotransmitters, or their mode of action remains uncertain ( $N, N$-dimethyltryptamine (DMT) is a good example of both (Ray, 2010; Wallach, 2009)), thus the specific systems that mediate the induction of synaesthesia may be difficult to identify. However, a neurochemical taxonomy of action may be possible once more is known about the action of these chemicals and the specific types and features of synaesthesia they induce (Luke et al., 2012).

A wide range of pharmacological agents, especially those termed psychedelic, have been reported to induce synaesthetic experience, extending back to the earliest subjective accounts by scientists and explorers using mescaline (Ellis, 1898), LSD (Hofmann, 1983), and psilocybin (Wasson, 1978). A recent survey (Luke et al., 2012) corroborates the wide range of substances reported to induce synaesthesia and suggests that the prevalence of this experience among those using these substances is higher with certain classes of psychoactive substances than others, particularly the serotonergic tryptamines (e.g., LSD, psilocybin), then the largely serotonergic substituted phenethylamines (mescaline, 2CB), then the glutamatergic dissociatives (dextromethorphan, ketamine), and then other drugs to a lesser extent. Here we review the few experimental studies that have investigated the induction of synaesthesia using pharmacological agents and then describe other studies that have used questionnaire and survey methodologies.

Despite there being a long history of subjective reports of spontaneous experiences, direct experimental research of chemically induced synaesthesia is sparse and, until very recently, has not been conducted since the 1960s when prohibition effectively curtailed psychedelic research with humans for the next 30-40 years. In one of the first systematic studies (Simpson and 
Terhune, Luke, \& Cohen Kadosh, 2017, chapter in Sensory Blending: On synaesthesia and other phenomena

McKellar, 1955), two non-synaesthete controls (the authors) and two congenital synaesthetes were each given four doses $(0.3-0.5 \mathrm{mg})$ of mescaline on different occasions and presented with an array of possible inducer stimuli throughout the course of their drug-induced altered state (up to 12 hours). The experimenters recorded any synaesthetic impressions but did not attempt to train or repeat experiences. Participants collectively reported eight distinct types of novel synaesthesia; inducers varied considerably, whereas the concurrent was typically visual, with the most common type being auditory-visual, which was experienced by each participant in at least one session. The single congenital synaesthete who had multiple types of synaesthesia also reported enhancement of their ordinary auditory-tactile and visual-tactile synaesthesias. The researchers correctly predicted that mescaline would produce novel variants; however, given their roles as experimenters, the controls were not blind, or impartial, to the aims of the study. This study has a number of clear limitations, in particular the lack of measures of consistency and/or automaticity of inducer-concurrent associations, but points to the potential viability of experimentally inducing synaesthesia. It also suggests that the same pharmacological agents that induce synaesthesia in non-synaesthetes enhance synaesthesia in congenital synaesthetes, which was also found in a recent survey (Luke et al., 2012).

A second experimental study used a more rigorous approach and compared a range of different substances in the induction of synaesthesia (Hartman and Hollister, 1963). Hartman and Hollister administered mescaline, psilocybin, and LSD a week apart to eighteen participants who were blind to the type of drug. Participants listened to sixteen pure sonic tones at four set frequencies (between 500 and $4000 \mathrm{~Hz}$ ) at relatively equal amplitudes at baseline and following the administration of each drug. Compared to baseline, the participants experienced significantly more colours and other visual effects (brightening of the visual field, shattering of patterns, and 
Terhune, Luke, \& Cohen Kadosh, 2017, chapter in Sensory Blending: On synaesthesia and other phenomena

patterning of form) during the presentation of pure tones whilst under the influence of both LSD and mescaline. Psilocybin was associated with a non-significant increase in these experiences. This study expands upon the study of Simpson and McKellar (1955) by showing the induction of auditory-visual synaesthesia with controlled stimuli. It further highlights the role of serotonin agonists in the induction of synaesthesia, although the inclusion of a placebo would have strengthened the design. Recent survey research (Luke et al., 2012) similarly found LSD to be the most prevalent inducer of synesthesia as a percentage of those using the drug (55 per cent).

A number of studies have similarly administered psychedelic substances to examine neurocognitive processes more generally and have used altered-states questionnaires that include items pertaining to synaesthesia. These approaches are clearly less rigorous than experimental designs, but can potentially lend insights into the induction of synaesthesia, particularly when they reveal convergent effects. That such scales include synaesthesia is indicative of its consideration as an ordinary, if not relatively infrequent, part of many altered states of consciousness (Dittrich and Scharfetter, 1987), as supported by the correlation between the synaesthesia subscale and all of the subscales of the Altered State of Consciousness Rating Scale (Studerus, Gamma, and Vollenweider, 2010) for psilocybin participants, ranging from elementary imagery, complex imagery, and experience of unity at one end to anxiety at the other.

Findings from studies employing a questionnaire approach highlight a wide range of pharmacological agents in the induction of synaesthesia. These studies suggest that the incidence of auditory-visual synaesthesia escalates linearly with psilocybin dosage (Griffiths et al., 2011; Studerus, 2013), and that reports of auditory-visual synaesthesia occur with decreasing prevalence from relatively equal doses of psilocybin (37 per cent), ketamine (27 per cent), and MDMA (10 per cent), as supported by prevalence figures from a survey of recreational users 
Terhune, Luke, \& Cohen Kadosh, 2017, chapter in Sensory Blending: On synaesthesia and other phenomena

(Luke et al., 2012). Reports of auditory-visual synaesthesia with psilocybin are also evident from different laboratories (Carhart-Harris et al., 2011), although prevalence rates are lower (11 per cent). Laboratory studies of other psychedelic substances in humans also report the subjective experience of synaesthesia, such as auditory-visual synaesthesia with ayahuasca, evident in 28 per cent of participants (Riba, Anderer, Jane, Saletu, and Barbanoj, 2004), and with Salvia divinorum, which reportedly induced visual-somatic synaesthesia in 57 per cent of participants (Addy, 2010). Interestingly, the largest such laboratory database (Studerus, 2013), comprising 261 participants and 409 psilocybin administrations, indicates that induced auditory-visual synaesthesia is strongly predicted by drug dosage and the Tellegen Absorption Scale (Tellegen and Atkinson, 1974), and weakly predicted by alcohol consumption, and under-the-influence self-reports of sociability, emotional excitability, and activity. This finding is notable for two reasons. First, it identifies a possible moderating factor of individual differences in susceptibility to experiencing synaesthesia under the influence of psilocybin. Second, the construct of absorption is indiscriminable from that of fantasy-proneness (Rhue and Lynn, 1989) and the fantasizing component of empathy has been recently shown to be elevated in congenital synaesthesia (Banissy et al., 2013). This suggests that individuals who have a cognitiveperceptual personality profile similar to that of congenital synaesthetes may be more susceptible to drug-induced synaesthesia.

Collectively, these and other studies suggest that LSD and other serotonin agonists reliably produce spontaneous synaesthesia-like experiences (Luke and Terhune, 2013). Despite these promising, albeit preliminary, results, the available studies on drug-induced synaesthesia are severely methodologically limited. For example, none of the research studies of this phenomenon have used double-blind methods, placebo controls, or established behavioural markers of 
Terhune, Luke, \& Cohen Kadosh, 2017, chapter in Sensory Blending: On synaesthesia and other phenomena

synaesthesia. To address this lacuna in contemporary knowledge, we recently conducted a double-blind placebo-controlled study investigating the effect of LSD on markers of synaesthesia (conscious accessibility, and inducer-concurrent consistency and specificity) in non-synaesthetes (Terhune, Luke, Kaelen, Bolstridge, Feilding, Nutt, Carhart-Harris, and Ward, 2016). After administration of placebo and LSD on separate days, participants rated the extent to which graphemes and sounds elicited conscious experience of colours and selected their first colour associations for these stimuli. Crucially, participants did not differ across the two conditions in the accessibility of colour concurrents, and their grapheme-colour associations did not differ in consistency or specificity. However, participants did report more spontaneous synaesthesia-like experiences in the LSD condition relative to the placebo condition. Although preliminary, this study clearly challenges the proposal that LSD produces genuine synaesthesia, as least according to the criteria by which we verify the occurrence of congenital synaesthesia.

Experimental and survey data, while sparse, indicate that spontaneous pharmacologically mediated synaesthesia is relatively reliable and widespread, especially with psychedelic agents (Luke et al., 2012; Luke and Terhune, 2013). Despite some researchers emphasizing the similarity between drug-induced and congenital synaesthesia in terms of their vividness, memorability, and emotionality (e.g., Cytowic, 1993; Cytowic, 2002; Cytowic and Eagleman, 2009), others have argued that they diverge in a number of important respects. Hubbard and Ramachandran $(2003,2005)$, for instance, maintain that pharmacologically induced synaesthesia lacks the specificity of congenital synaesthesia and is also more complex than the simple inducer-concurrent associations experienced by congenital synaesthetes. Our preliminary data supports this argument (Terhune et al., 2016). However, it could be argued that the complexity of psychedelic synaesthesia, while certainly reported by some (e.g., Klïver, 1966), is not obligatory 
Terhune, Luke, \& Cohen Kadosh, 2017, chapter in Sensory Blending: On synaesthesia and other phenomena

(e.g., Simpson and McKellar, 1955). Sinke et al. (2012) further suggest that drug-induced synaesthesia lacks the automaticity and consistency of congenital synaesthesia, citing an older study which explored the consistency of sound-colour synaesthesia with mescaline (Beringer, 1927). Indeed, our study similarly found no effect of LSD on grapheme- or sound-colour consistency (Terhune et al., 2016). However, in one case, melatonin-induced grapheme-colour synaesthesia was shown to display consistency via a texture segregation behavioural test (Brang and Ramachandran, 2008). Interestingly, there is suggestive evidence that mescaline-induced synaesthesia can reproduce individual differences in synaesthetes. Klüver (1966: 93), for instance, notes that on mescaline, an "auditory stimulus may give rise to a sensation of color in some subjects, but in others the color, e.g., purple, is not actually seen. Instead, the subject experiences a 'feeling like purple' or a feeling 'as if purple"'. It remains unclear whether this reflects broader individual differences in psychedelic experiences or something that is specific to the induction of synaesthesia.

A final difference between congenital and drug-induced synaesthesias may be in the types of inducers and concurrents. Sinke et al. (2012) note that congenital synaesthesias typically have graphemes as inducers, whereas drug-induced synaesthesias do not. Although it's true that graphemes are frequent inducers, there is evidence that music and sound stimuli function as inducers in more than 25 per cent of cases of congenital synaesthesia (Hochel and Milắn, 2008). This is notable because sound-colour (or sound-visual) synaesthesia appears to be the most frequently observed type of drug-induced synaesthesia (Klüiver, 1966; Luke et al., 2012; Pahnke and Richards, 1966; Simpson and McKellar, 1955; Sinke et al., 2012; for a review, see Luke and Terhune, 2013). Furthermore, approximately 1 per cent of recreational tryptamine psychedelic (e.g., psilocybin, LSD) users report spontaneous grapheme-colour synaesthesia (Luke et al., 
Terhune, Luke, \& Cohen Kadosh, 2017, chapter in Sensory Blending: On synaesthesia and other phenomena

2012) and at least one case of verified drug-induced grapheme-colour synaesthesia has been reported (Brang and Ramachandran, 2008). Moreover, the patterns of different synaesthesia types across congenital and drug-induced synaesthesias are most likely artefactual of the context in which people consume drugs. Specifically, during the consumption of psychedelic drugs, people are more likely to listen to music than to read and thus the prevalence rates of soundcolour and grapheme-colour synaesthesias under the influence of such drugs are very likely to be inflated and deflated, respectively.

Discerning the relationship between drug-induced and congenital synaesthesias is crucial because it may help us to identify the neurochemical markers of congenital synaesthesia and to discriminate between competing theories of synaesthesia. Disinhibition theories propose that the experience of synaesthesia is normally suppressed (Cohen Kadosh and Henik, 2007; Eagleman and Goodale, 2009; Grossenbacher, 1997) and thus these accounts can easily accommodate druginduced synaesthesias. Disinhibition theories predict a reduction in GABA in visual cortex as a possible neurochemical mechanism underlying congenital synaesthesia. To date, no study has explicitly examined the influence of GABAergic agonists or antagonists on the experience of synaesthesia, although one study found that synaesthetes and controls don't differ in visual cortex GABA levels (Terhune et al., 2015), and thus there is as yet no direct evidence bearing on the implications of drug-induced synaesthesia for disinhibition theories of synaesthesia. Conversely, cross-activation theory suggests that congenital and drug-induced synaesthesias occur through disparate mechanisms (Hubbard et al., 2011).

Whether there are multiple aetiologies for synaesthesia or not, current theorizing proposes that serotonin-2A subtype agonism is fundamental to drug-induced synaesthesia (Brang and Ramachandran, 2008). In support of the $5 \mathrm{HT}_{2 \mathrm{~A}}$ hypothesis, Brang and Ramachandran (2008) 
note that LSD largely operates via $5 \mathrm{HT}_{2 \mathrm{~A}}$ agonism, that the presumed $5 \mathrm{HT}_{2 \mathrm{~A}}$ inhibitors Prozac and Wellbutrin have been shown to inhibit congenital synaesthesia in case studies, and that melatonin was able to induce genuine grapheme-colour synaesthesia like a chemical switch, possibly via $5 \mathrm{HT}_{1}$ inhibition and subsequent $5 \mathrm{HT}_{2 \mathrm{~A}}$ disinhibition. However, whole-gene linkage scan and family-based linkage analysis studies have as yet not found evidence for a $5 \mathrm{HT}_{2 \mathrm{~A}^{-}}$ linked gene for synaesthesia, perhaps due to the low power of the studies or a polygenic aetiology (Brang and Ramachandran, 2011). Nevertheless, recent survey research (Luke et al., 2012) lends some support to the $5 \mathrm{HT}_{2 \mathrm{~A}}$ agonism hypothesis, as serotonergic tryptamines (e.g., LSD, psilocybin) and the largely serotonergic substituted phenethylamines (mescaline, 2CB) were shown to be the most prevalent inducers of synaesthesia, although other classes of drugs were also reported to induce synaesthesia too, albeit to a lesser extent. Nevertheless, the psychedelic Salvia divinorum, which has no known serotonergic action, but known kappa opioid receptor activation only (Ray, 2010), was reported to induce synaesthesia with moderate prevalence in this survey (33 per cent) and in a laboratory study (57 per cent) (Addy, 2010). These results appear to challenge the serotoninergic hypothesis, but it is possible that kappa receptors regulate the serotonin system (Bruchas et al., 2011), giving rise to secondary serotonergic effects. Furthermore, not only do serotonergic psychedelics have the highest prevalence rates for inducing synaesthesias among non-synaesthetes, they also have the greatest tendency of any class of substances to enhance the existing synaesthesia of congenital synaesthetes (Luke et al., 2012), although caution is urged over the interpretation of self-report data from a self-selecting sample. Cumulatively, these results implicate serotonin in both the induction of synaesthesia in non-synaesthetes and its amelioration in congenital synaesthetes, but 
Terhune, Luke, \& Cohen Kadosh, 2017, chapter in Sensory Blending: On synaesthesia and other phenomena

further research is clearly needed to corroborate these results with more rigorous experimental designs.

In summary, using pharmacological agents to induce and inhibit synaesthesia has advantages in that it can elicit effects quickly and with a large sample of people, and it also has the potential to illuminate the neurotransmitter systems involved in at least some subtypes of synaesthesia. However, little research has actually been conducted along these lines as yet, so current understanding is extremely limited and there is much to be learned. Our recent research suggests that LSD-induced synaesthesia does not meet standard criteria for synaesthesia (conscious accessibility and inducer-concurrent consistency and specificity) and thereby potentially call into question this line of research (Terhune et al., 2016). By contrast, we are unaware of any investigation into the automaticity of inducer-concurrent associations.

Nevertheless, as we noted earlier in this chapter, it may not be particularly meaningful to apply the criterion of consistency to early-stage synaesthesias. Given the relative paucity of data, we believe it remains premature to draw firm conclusions regarding the authenticity of drug-induced synaesthesias. Working with psychoactive substances can be challenging, but a growing interest in the neurobiological action of psychedelics makes this research more feasible now than previously. Nevertheless, despite group trends being somewhat well mapped, psychedelic agents produce largely unpredictable altered states of consciousness within individuals and effects other than synaesthesia will be produced that will need to be addressed. Despite these challenges, we believe that the use of pharmacological agents will be useful in helping to understand the neurobiology of synaesthesia.

\subsection{Comparing methods}


Terhune, Luke, \& Cohen Kadosh, 2017, chapter in Sensory Blending: On synaesthesia and other phenomena

Here we briefly contrast the different methods of inducing synaesthesia. The studies conducted to date overwhelmingly demonstrate that posthypnotic suggestion is a more robust method for the experimental induction of synaesthesia than training approaches. Training is time-intensive (Bor et al., 2014; but see Kusnir and Knut, 2012), it does not reliably reproduce behavioural or phenomenological markers of synaesthesia, and it does not replicate the physiological and neurophysiological concomitants of synaesthesia. Furthermore, training has yet to reproduce individual differences in synaesthetic experience (Dixon et al., 2004; Ward et al., 2007). In contrast, posthypnotic suggestion can reliably reproduce the behavioural and phenomenological markers of synaesthesia, as well as individual differences therein, and it is effective in a relatively short time period. A further benefit of posthypnotic suggestion is that it can be used to modulate synaesthesia phenomenology in highly suggestible synaesthetes (see also Terhune, Cardeña,a, and Lindgren, 2010). We find it highly unlikely that training could accomplish this.

Nevertheless, the use of posthypnotic suggestion for the induction and modulation of synaesthesia has its limitations whilst training has other strengths. The posthypnotic induction of synaesthesia is hampered by the fact that posthypnotic suggestion is only reliably effective in highly suggestible individuals, who comprise a small minority of the population (Laurence et al., 2008), and extensive screening is required to identify these individuals (Woody and Barnier, 2008). We maintain that this explains failures to induce synaesthesia with suggestion (Anderson et al., 2014). Training, on the other hand, can be done with anyone, although there clearly seem to be important individual differences (Bor et al., 2014; Colizoli et al., 2012; Kusnir and Thut, 2012). Moreover, one might argue that training is more comparable to the real-life process by which inducer-concurrent associations are initially formed and consolidate over time. Posthypnotic suggestion, in contrast, does not present itself as a valuable method for studying the 
learning mechanisms underlying synaesthesia. Thus, whilst the available evidence reliably demonstrates that posthypnotic suggestion is a superior method for the induction of synaesthesia, training is clearly a better model for learning in synaesthesia. We hope to see both methods strengthened with further research. For instance, it would be valuable to undertake more rigorous screening of participants to exploit individual differences and identify participants who are most likely to develop strong associations from training, such as relatives of synaesthetes (Colizoli et al., 2016) or individuals with strong imagery (Bor et al., 2014).

In certain respects, psychedelic synaesthesias may present the closest model of genuine congenital synaesthesia. Synaesthesias experienced after the consumption of pharmacological agents are relatively common and thus this approach is likely to be more reliable than posthypnotic suggestion and more amenable to broad research. As in congenital synaesthesia, drug-induced synaesthesias appear to emerge relatively spontaneously. This is in contrast with training methods, which require an experimental manipulation to elicit concurrent experiences (posthypnotic suggestion) or grapheme-colour associations (training). Like posthypnotic suggestion, but unlike training, the induction of synaesthesia using pharmacological agents can also reproduce conscious awareness of synaesthetic concurrents. Taken together, these strengths suggest that this approach is the most viable for large-scale studies of induced synaesthesia. Drug-induced synaesthesias may potentially resemble early-stage synaesthesias that have not yet undergone consolidation. In addition, drug-induced synaesthesias provide a clearer framework for studying the neurochemical mechanisms of synaesthesia than do posthypnotic suggestion and training. However, despite the value of this approach, it does not afford the ability to carefully manipulate the phenomenology of synaesthesia, as does posthypnotic suggestion. Indeed, it has been argued that drug-induced synaesthesias are pervasive, inconsistent (Sinke et al., 2012), and 
Terhune, Luke, \& Cohen Kadosh, 2017, chapter in Sensory Blending: On synaesthesia and other phenomena

non-specific (Hubbard et al., 2011), and thus may not be very amenable to research studies concerned with the characteristics and mechanisms of a specific form of synaesthesia. Ways of leveraging the strengths of this approach whilst circumventing these limitations would be to stratify participants by type of previous self-reported drug-induced synaesthesia and to explore the possibility of training inducer-concurrent associations after the consumption of pharmacological agents. Further exploring the combination of hypnosis and psychoactive substances could prove illuminating in experimental conditions, especially in light of the supposedly increased suggestibility of the psychedelic state (Sjoberg and Hollister, 1965; Carhart-Harris, Kaelen, Whalley, Bolstridge, Feilding, and Nutt, 2015) and the possibility of reinducing such drug states via hypnotic suggestion (Hastings, 2006).

\subsection{Implications and conclusions}

The induction of synaesthesia using the methods described in this chapter has a number of significant implications. First, these approaches present potentially viable methods for experimental analogues of synaesthesia that can be used to aid our understanding of the cognitive and neural mechanisms of this condition. We hope to see further research exploring the induction of synaesthesia using these methods, but also research that uses such methods to answer questions about synaesthesia (Luke et al., 2012; Terhune and Cohen Kadosh, 2012b). The methods described here also have clear implications for the criteria by which synaesthesia is defined. Although none of the methods has been shown to meet all of the criteria of synaesthesia that we outlined at the beginning of this chapter, we are very confident that posthypnotic suggestion will be able to reproduce all of the hallmark features of synaesthesia. In turn, such research will have important implications regarding the utility of such criteria. 
A diverse array of studies have explored the possibility that synaesthesia can be induced. These have included training, posthypnotic suggestion, and the administration of pharmacological agents. The foregoing review suggests that training can produce graphemecolour associations, although the magnitude of these associations varies, as does the extent to which they impact different behavioural markers of synaesthesia. Crucially, training studies have so far been unable to reliably produce colour photisms although preliminary research is encouraging. In contrast, posthypnotic suggestion is able to elicit both the behavioural and phenomenological markers of synaesthesia, as well as individual differences therein. Pharmacological agents have been shown to elicit the phenomenological features of synaesthesia, but the extent to which they reproduce the behavioural markers of this condition is unclear and preliminary research indicates that drug-induced synaesthesia is unlikely to meet standard criteria for synaesthesia. Multiple studies have shown that training does not reproduce the physiological and neural signatures of synaesthesia, whereas the impact of posthypnotic suggestion and pharmacological agents on these signatures has yet to be investigated. These different methods have their individual limitations but each can make unique contributions to our understanding of synaesthesia.

\section{Acknowledgements}

D.B.T. is supported by a Marie Curie Intra-European Fellowship within the 7th European Community Framework Programme and Bial Foundation bursary 344/14. D.P.L. is supported by University of Greenwich RAE grant R09469. R.C.K. is supported by the Wellcome Trust (WT88378).

\section{References}


Terhune, Luke, \& Cohen Kadosh, 2017, chapter in Sensory Blending: On synaesthesia and other phenomena

Addy, P. H. (2010). That deep internal voice: Controlled administration of Salvia divinorum. Unpublished PhD thesis, Institute of Transpersonal Psychology, Palo Alto, CA.

Alstadhaug, K. B., \& Benjaminsen, E. (2010). Synesthesia and migraine: Case report. BMC Neurology, 10. doi: 10.1186/1471-2377-10-121

Anderson, H.P., Seth, A.K., Dienes, Z., \& Ward, J. (2014). Can grapheme-color synesthesia be induced by hypnosis? Frontiers in Human Neuroscience, 8, 220. doi:

$10.3389 /$ fnhum.2014.00220

Banissy, M. J., Holle, H., Cassell, J., Annett, L., Tsakanikos, E., Walsh, V., Ward, J. et al. (2013). Personality traits in people with synaesthesia: Do synaesthetes have an atypical personality profile? Personality and Individual Differences, 54, 828-31. doi: 10.1016/j.paid.2012.12.018

Banissy, M.J., Tester, V., Muggleton, N.G., Janik, A.B., Davenport, A., Franklin, A., Walsh, V., \& Ward, J. (2013). Synesthesia for color is linked to improved color perception but reduced motion perception. Psychological Science, 24, 2390-2397. doi: $10.1177 / 0956797613492424$

Banissy, M. J., Walsh, V., \& Ward, J. (2009). Enhanced sensory perception in synaesthesia. Experimental Brain Research, 196, 565-71. doi: 10.1007/s00221-009-1888-0

Barnett, K. J., Foxe, J. J., Molholm, S., Kelly, S. P., Shalgi, S., Mitchell, K. J., \& Newell, F. N. (2008). Differences in early sensory-perceptual processing in synesthesia: A visual evoked potential study. NeuroImage, 43, 605-13. doi: 10.1016/j.neuroimage.2008.07.028

Beringer, K. (1927). Der Meskalinrausch. Berlin: Springer.

Bor, D., Rothen, N., Schwartzman, D.J., Clayton, S., \& Seth, A.K. (2014). Adults can be trained to acquire synesthetic experiences. Scientific Reports, 4, 7089. doi: 
10.1038/srep07089.

Bowers, K. S. (1981). Do the Stanford Scales tap the "classic suggestion effect"? International Journal of Clinical and Experimental Hypnosis, 29, 42-53.

Brang, D., Hubbard, E. M., Coulson, S., Huang, M., \& Ramachandran, V. S. (2010).

Magnetoencephalography reveals early activation of V4 in grapheme-color synesthesia. Neuroimage, 53, 268-74.

Brang, D., \& Ramachandran, V. S. (2008). Psychopharmacology of synesthesia: The role of serotonin S2a receptor activation. Medical Hypotheses, 70, 903-4.

Brang, D., \& Ramachandran, V. S. (2011). Survival of the synesthesia gene: Why do people hear colors and taste words? PLoS Biology, 9, e1001205. doi: 10.1371/journal.pbio.1001205

Brown, R. J., Antonova, E., Langley, A., \& Oakley, D. A. (2001). The effects of absorption and reduced critical thought on suggestibility in an hypnotic context. Contemporary Hypnosis, $18,62-72$.

Bruchas, M. R., Schindler, A. G., Shankar, H., Messinger, D. I., Miyatake, M., Land, B. B., Chavkin, C. et al. (2011). Selective p38alpha MAPK deletion in serotonergic neurons produces stress resilience in models of depression and addiction. Neuron, 71, 498-511. doi: 10.1016/j.neuron.2011.06.011

Cappelletti, M., Gessaroli, E., Hithersay, R., Mitolo, M., Didino, D., Kanai, R., Cohen Kadosh, R., \& Walsh, V. (2013). Transfer of cognitive training across magnitude dimensions achieved with concurrent brain stimulation of the parietal lobe. Journal of Neuroscience, 33, 14899-14907. doi: 10.1523/JNEUROSCI.1692-13.2013

Carhart-Harris, R. L., Erritzoe, D., Williams, T., Stone, J. M., Reed, L. J., Colasanti, A., Nutt, D. J. et al. (2012). Neural correlates of the psychedelic state as determined by fMRI studies 
Terhune, Luke, \& Cohen Kadosh, 2017, chapter in Sensory Blending: On synaesthesia and other phenomena

with psilocybin. Proceedings of the National Academy of Sciences of the United States of America, 109, 2138-43. doi: 10.1073/pnas.1119598109

Carhart-Harris, R.L., Kaelen, M., Whalley, M.G., Bolstridge, M., Feilding, A., \& Nutt, D.J. (2015). LSD enhances suggestibility in healthy volunteers. Psychopharmacology (Berlin), 232, 785-794. doi: 10.1007/s00213-014-3714-Z

Carhart-Harris, R. L., Williams, T. M., Sessa, B., Tyacke, R. J., Rich, A. S., Feilding, A., \& Nutt, D. J. (2011). The administration of psilocybin to healthy, hallucinogen-experienced volunteers in a mock-functional magnetic resonance imaging environment: A preliminary investigation of tolerability. Journal of Psychopharmacology, 25, 1562-7. doi:

\section{$10.1177 / 0269881110367445$}

Cohen, M.X., Weidacker, K., Tankink, J., Scholte, H.S., \& Rouw, R. (2015). Grapheme-color synesthesia subtypes: Stable individual differences reflected in posterior alpha-band oscillations. Cognitive Neuroscience, 6, 56-67. doi: 10.1080/17588928.2015.1017450

Cohen Kadosh, R., \& Henik, A. (2007). Can synaesthesia research inform cognitive science? Trends in Cognitive Sciences, 11, 177-84.

Cohen Kadosh, R., Henik, A., Catena, A., Walsh, V., \& Fuentes, L. J. (2009). Induced crossmodal synaesthetic experience without abnormal neuronal connections. Psychological Science, 20, 258-65. doi: 10.1111/j.1467-9280.2009.02286.x

Cohen Kadosh, R., Sagiv, N., Linden, D. E., Robertson, L. C., Elinger, G., \& Henik, A. (2005). When blue is larger than red: Colors influence numerical cognition in synesthesia. Journal of Cognitive Neuroscience, 17, 1766-73. doi: 10.1162/089892905774589181

Cohen Kadosh, R., \& Walsh, V. (2008). Synaesthesia and cortical connections: Cause or correlation? Trends in Neurosciences, 31, 549-50. 
Terhune, Luke, \& Cohen Kadosh, 2017, chapter in Sensory Blending: On synaesthesia and other phenomena

Cojan, Y., Waber, L., Schwartz, S., Rossier, L., Forster, A., \& Vuilleumier, P. (2009). The brain under self-control: Modulation of inhibitory and monitoring cortical networks during hypnotic paralysis. Neuron, 62, 862-75. doi: 10.1016/j.neuron.2009.05.021

Colizoli, O., Murre, J. M., \& Rouw, R. (2012). Pseudo-synesthesia through reading books with colored letters. PloS One, 7, e39799. doi: 10.1371/journal.pone.0039799

Colizoli, O., Murre, J.M., \& Rouw, R. (2014). Defining (trained) grapheme-color synesthesia. Frontiers in Human Neuroscience 8, 368. doi: 10.3389/fnhum.2014.00368

Colizoli, O., Murre, J.M., Scholte, H.S., van Es, D.M., Knapen, T., \& Rouw, R. (2016). Visual cortex activity predicts subjective experience after reading books with colored letters.

Neuropsychologia, 88, 15-27. doi: 10.1016/j.neuropsychologia.2015.07.006

Cytowic, R. E. (1993). The man who tasted shapes: A bizarre medical mystery offers revolutionary insights into emotions, reasoning, and consciousness. New York: Tarcher/Putnam.

Cytowic, R. E. (2002). Synesthesia: A union of the senses. Cambridge, MA: MIT Press.

Cytowic, R. E., \& Eagleman, D. M. (2009). Wednesday is indigo blue: Discovering the brain of synesthesia. Cambridge, MA: MIT Press.

Deroy, O., \& Spence, C. (2013). Why we are not all synesthetes (not even weakly so). Psychonomic Bulletin \& Review, 20, 643-664. doi: 10.3758/s13423-013-0387-2

Dienes, Z., \& Hutton, S. (2013). Understanding hypnosis metacognitively: rTMS applied to DLPFC increases hypnotic suggestibility. Cortex, 49, 386-392. Doi:

10.1016/j.cortex.2012.07.009 
Terhune, Luke, \& Cohen Kadosh, 2017, chapter in Sensory Blending: On synaesthesia and other phenomena

Dittrich, A., \& Scharfetter, C. (Eds.). (1987). Ethnopsychotherapie: Psychotherapie mittels außergewöhnlicher Bewußtseinszustände in westlichen und indigenen Kulturen. Stuttgart: Enke.

Dixon, M. J., Smilek, D., Cudahy, C., \& Merikle, P. M. (2000). Five plus two equals yellow. Nature, 406, 365. doi: 10.1038/35019148

Dixon, M. J., Smilek, D., \& Merikle, P. M. (2004). Not all synaesthetes are created equal: Projector versus associator synaesthetes. Cognitive Affective and Behavioral Neuroscience, $4,335-43$.

Eagleman, D. M., \& Goodale, M. A. (2009). Why color synesthesia involves more than color. Trends in Cognitive Sciences, 13, 288-92.

Egner, T., Jamieson, G., \& Gruzelier, J. (2005). Hypnosis decouples cognitive control from conflict monitoring processes of the frontal lobe. Neuroimage, 27, 969-78.

Egner, T., \& Raz, A. (2007). Cognitive control processes and hypnosis. In G. A. Jamieson (Ed.), Hypnosis and conscious states: The cognitive neuroscience perspective (pp. 29-50). Oxford, UK: Oxford University Press.

Elias, L. J., Saucier, D. M., Hardie, C., \& Sarty, G. E. (2003). Dissociating semantic and perceptual components of synaesthesia: Behavioural and functional neuroanatomical investigations. Cognitive Brain Research, 16, 232-7. doi: 10.1016/S0926-6410(02)00278-1

Ellis, H. (1898). Mescal: A new artificial paradise. The Contemporary Review, 73.

Fertonani, A., Pirulli, C., \& Miniussi, C. (2011). Random noise stimulation improves neuroplasticity in perceptual learning. Journal of Neuroscience, 31, 15416-15423. doi: 10.1523/JNEUROSCI.2002-11.2011 
Terhune, Luke, \& Cohen Kadosh, 2017, chapter in Sensory Blending: On synaesthesia and other phenomena

Fornazzari, L., Fischer, C. E., Ringer, L., \& Schweizer, T. A. (2012). "Blue is music to my ears": Multimodal synesthesias after a thalamic stroke. Neurocase, 18, 318-22. doi: $10.1080 / 13554794.2011 .608362$

Gebuis, T., Nijboer, T. C., \& Van der Smagt, M. J. (2009). Multiple dimensions in bidirectional synesthesia. European Journal of Neuroscience, 29, 1703-10.

Griffiths, R. R., Johnson, M. W., Richards, W. A., Richards, B. D., McCann, U., \& Jesse, R. (2011). Psilocybin occasioned mystical-type experiences: Immediate and persisting doserelated effects. Psychopharmacology, 218, 649-65. doi: 10.1007/s00213-011-2358-5

Grossenbacher, P. G. (1997). Perception and sensory information in synaesthetic experience. In S. Baron-Cohen \& J. E. Harrison (Eds.), Synaesthesia: Classic and contemporary readings (pp. 148-72). Oxford: Blackwell.

Hancock, P. (2006). Monozygotic twins' colour-number association: A case study. Cortex, 42, $147-50$.

Hartman, A. M., \& Hollister, L. E. (1963). Effect of mescaline, lysergic acid diethylamide and psilocybin on color perception. Psychopharmacologia, 4, 441-51.

Hastings, A. (2006). An extended nondrug MDMA-like experience evoked through posthypnotic suggestion. Journal of Psychoactive Drugs, 38, 273-83. doi: $10.1080 / 02791072.2006 .10399853$

Hilgard, E. R. (1965). Hypnotic susceptibility. New York: Harcourt, Brace \& World.

Hochel, M., \& Milắn, E. G. (2008). Synaesthesia: The existing state of affairs. Cognitive Neuropsychology, 25, 93-117.

Hofmann, A. (1983). LSD my problem child: Reflections on sacred drugs, mysticism, and science. Los Angeles: Jeremy P. Tarcher. 
Terhune, Luke, \& Cohen Kadosh, 2017, chapter in Sensory Blending: On synaesthesia and other phenomena

Hubbard, E. M. (2007). A real red-letter day. Nature Neuroscience, 10, 671-2.

Hubbard, E. M. (2011). The cross-activation theory at ten: Substantial growth, future challenge. Paper presented at the UK Synaesthesia Association, London, UK.

Hubbard, E. M., Arman, A. C., Ramachandran, V. S., \& Boynton, G. M. (2005). Individual differences among grapheme-color synesthetes: brain-behavior correlations. Neuron, 45, $975-85$.

Hubbard, E. M., Brang, D., \& Ramachandran, V. S. (2011). The cross-activation theory at 10. Journal of Neuropsychology, 5, 152-77. doi: 10.1111/j.1748-6653.2011.02014.x

Hubbard, E. M., \& Ramachandran, V. S. (2003). Refining the experimental lever: A reply to Shanon \& Pribram. Journal of Consciousness Studies, 9, 77-84.

Hubbard, E. M., \& Ramachandran, V. S. (2005). Neurocognitive mechanisms of synesthesia. Neuron, 48, 509-20.

Hupé, J. M., Bordier, C., \& Dojat, M. (2011). The neural bases of grapheme-color synesthesia are not localized in real color-sensitive areas. Cerebral Cortex. doi: 10.1093/cercor/bhr236

Klüver, H. (1966). Mescal and mechanisms of hallucinations. Chicago, IL: University of Chicago Press.

Kosslyn, S. M., Thompson, W. L., Costantini-Ferrando, M. F., Alpert, N. M., \& Spiegel, D. (2000). Hypnotic visual illusion alters color processing in the brain. American Journal of Psychiatry, 157, 1279-84.

Krause, B., \& Cohen Kadosh, R. (2013). Can transcranial electrical stimulation improve learning difficulties in atypical brain development? A future possibility for cognitive training. Developmental Cognitive Neuroscience, 6(0), 176-194. doi:

http://dx.doi.org/10.1016/j.den.2013.04.001 
Terhune, Luke, \& Cohen Kadosh, 2017, chapter in Sensory Blending: On synaesthesia and other phenomena

Kusnir, F., \& Thut, G. (2012). Formation of automatic letter-colour associations in nonsynaesthetes through likelihood manipulation of letter-colour pairings. Neuropsychologia, 50, 3641-52. doi: 10.1016/j.neuropsychologia.2012.09.032

Laurence, J.-R., Beaulieu-Prévost, D., \& du Chêné, T. (2008). Measuring and understanding individual differences in hypnotizability. In M. R. Nash \& A. Barnier (Eds.), The Oxford handbook of hypnosis (pp. 225-53). Oxford: Oxford University Press.

Lee, H.-M., \& Roth, B. L. (2012). Hallucinogen actions on human brain revealed. Proceedings of the National Academy of Sciences of the United States of America, 109.

Looi, C.Y., Duta, M., Brem, A.K., Huber, S., Nuerk, H.C., \& Cohen Kadosh, R. (2016). Combining brain stimulation and video game to promote long-term transfer of learning and cognitive enhancement. Scientific Reports, 6, 22003. doi: 10.1038/srep22003

Ludwig, V. U., Adachi, I., \& Matsuzawa, T. (2011). Visuoauditory mappings between high luminance and high pitch are shared by chimpanzees (Pan troglodytes) and humans. Proceedings of the National Academy of Sciences of the United States of America, 108, 20661-5. doi: 10.1073/pnas.1112605108

Luke, D.P., \& Terhune, D.B. (2013). The induction of synaesthesia with chemical agents: A systematic review. Fronters in Psychology, 4, 753. doi: 10.3389/fpsyg.2013.00753

Luke, D., Terhune, D. B., \& Friday, R. (2012). Psychedelic synaesthesia: Evidence for a serotonergic role in synaesthesia. Seeing and Perceiving, S1, 74.

Marcusson-Clavertz, D., Terhune, D. B., \& Cardeña, E. (2012). Individual differences and state effects on mind-wandering: Hypnotizability, dissociation, and sensory homogenization. Consciousness and Cognition, 21, 1097-108. doi: 10.1016/j.concog.2012.04.002 
Terhune, Luke, \& Cohen Kadosh, 2017, chapter in Sensory Blending: On synaesthesia and other phenomena

Mattingley, J. B. (2009). Attention, automaticity, and awareness in synesthesia. Ann N Y Acad Sci, 1156, 141-67.

Mattingley, J. B., Rich, A. N., Yelland, G., \& Bradshaw, J. L. (2001). Unconscious priming eliminates automatic binding of colour and alphanumeric form in synaesthesia. Nature, 410 , 580-2. doi: $10.1038 / 35069062$

McGeown, W. J., Venneri, A., Kirsch, I., Nocetti, L., Roberts, K., Foan, L., \& Mazzoni, G. (2012). Suggested visual hallucination without hypnosis enhances activity in visual areas of the brain. Consciousness and Cognition, 21, 100-16. doi: 10.1016/j.concog.2011.10.015

Meier, B., \& Rothen, N. (2007). When conditioned responses "fire back": Bidirectional crossactivation creates learning opportunities in synesthesia. Neuroscience, 147, 569-72.

Meier, B., \& Rothen, N. (2009). Training grapheme-colour associations produces a synaesthetic Stroop effect, but not a conditioned synaesthetic response. Neuropsychologia, 47, 1208-11. doi: 10.1016/j.neuropsychologia.2009.01.009

Mendelsohn, A., Chalamish, Y., Solomonovich, A., \& Dudai, Y. (2008). Mesmerizing memories: Brain substrates of episodic memory suppression in posthypnotic amnesia. Neuron, 57, 159-70. doi: 10.1016/j.neuron.2007.11.022

Nikolić, D., Jurgens, U. M., Rothen, N., Meier, B., \& Mroczko, A. (2011). Swimming-style synesthesia. Cortex, 47, 874-9. doi: 10.1016/j.cortex.2011.02.008

Nikolić, D., Lichti, P., \& Singer, W. (2007). Color opponency in synaesthetic experiences. Psychological Science, 18, 481-6.

Nunn, J. A., Gregory, L. J., Brammer, M., Williams, S. C., Parslow, D. M., Morgan, M. J., Gray, J. A. et al. (2002). Functional magnetic resonance imaging of synesthesia: Activation of V4/V8 by spoken words. Nature Neuroscience, 5, 371-5. 
Terhune, Luke, \& Cohen Kadosh, 2017, chapter in Sensory Blending: On synaesthesia and other phenomena

Oakley, D. A., \& Halligan, P. W. (2009). Hypnotic suggestion and cognitive neuroscience. Trends in Cognitive Sciences, 13, 264-70. doi: 10.1016/j.tics.2009.03.004

Pahnke, W. N., \& Richards, W. A. (1966). Implications of LSD and experimental mysticism. Journal of Religion and Health, 5, 175-208.

Raichle, M. E., MacLeod, A. M., Snyder, A. Z., Powers, W. J., Gusnard, D. A., \& Shulman, G. L. (2001). A default mode of brain function. Proceedings of the National Academy of Sciences of the United States of America, 98, 676-82. doi: 10.1073/pnas.98.2.676

Ray, T. S. (2010). Psychedelics and the human receptorome. PLoS One, 5, e9019. doi: 10.1371/journal.pone.0009019

Reis, J., Schambra, H.M., Cohen, L.G., Buch, E.R., Fritsch, B., Zarahn, E., Celnik, P.A., \& Krakauer, J.W. (2009). Noninvasive cortical stimulation enhances motor skill acquisition over multiple days through an effect on consolidation. Proceedings of the National Academy of Sciences of the United States of America, 106, 1590-1595. doi: 10.1073/pnas.0805413106

Rhue, J. W., \& Lynn, S. J. (1989). Fantasy proneness, hypnotizability, and absorption—a reexamination: A brief communication. International Journal of Clinical and Experimental Hypnosis, 37, 100-6.

Riba, J., Anderer, P., Jane, F., Saletu, B., \& Barbanoj, M. J. (2004). Effects of the South American psychoactive beverage ayahuasca on regional brain electrical activity in humans: A functional neuroimaging study using low-resolution electromagnetic tomography. Neuropsychobiology, 50, 89-101. doi: 10.1159/000077946

Ro, T., Farne, A., Johnson, R. M., Wedeen, V., Chu, Z., Wang, Z. J., Beauchamp, M. S. et al. (2007). Feeling sounds after a thalamic lesion. Annals of Neurology, 62, 433-41. doi: 10.1002/ana.21219 
Terhune, Luke, \& Cohen Kadosh, 2017, chapter in Sensory Blending: On synaesthesia and other phenomena

Rothen, N., \& Meier, B. (2014). Acquiring synaesthesia: insights from training studies. Frontiers in Human Neuroscience 8, 109. doi: 10.3389/fnhum.2014.00109

Rothen, N., Meier, B., \& Ward, J. (2012). Enhanced memory ability: Insights from synaesthesia. Neuroscience and Biobehavioral Reviews, 36, 1952-63. doi:

10.1016/j.neubiorev.2012.05.004

Rothen, N., Seth, A.K., Witzel, C., \& Ward, J. (2013). Diagnosing synaesthesia with online colour pickers: maximising sensitivity and specificity. Journal of Neuroscience Methods, 215, 156-160. doi: 10.1016/j.jneumeth.2013.02.009

Rothen, N., Wantz, A. L., \& Meier, B. (2011). Training synaesthesia. Perception, 40, 1248-50.

Simner, J. (2012). Defining synaesthesia. British Journal of Psychology, 103, 1-15. doi: $10.1348 / 000712610 X 528305$

Rouw, R., \& Scholte, H.S. (2010). Neural basis of individual differences in synesthetic experiences. Journal of Neuroscience, 30, 6205-6213. doi: 10.1523/JNEUROSCI.344409.2010

Simner, J., Harrold, J., Creed, H., Monro, L., \& Foulkes, L. (2009). Early detection of markers for synaesthesia in childhood populations. Brain, 132, 57-64.

Simner, J., \& Holenstein, E. (2007). Ordinal linguistic personification as a variant of synesthesia. Journal of Cognitive Neuroscience, 19, 694-703. doi:

10.1162/jocn.2007.19.4.694

Simner, J., Mulvenna, C., Sagiv, N., Tsakanikos, E., Witherby, S. A., Fraser, C., Ward, J. et al. (2006). Synaesthesia: The prevalence of atypical cross-modal experiences. Perception, 35, 1024-33. doi: $10.1068 / \mathrm{p} 5469$ 
Terhune, Luke, \& Cohen Kadosh, 2017, chapter in Sensory Blending: On synaesthesia and other phenomena

Simpson, L., \& McKellar, P. (1955). Types of synaesthesia. Journal of Mental Science, 101, $141-7$.

Sinke, C., Halpern, J. H., Zedler, M., Neufeld, J., Emrich, H. M., \& Passie, T. (2012). Genuine and drug-induced synesthesia: A comparison. Consciousness and Cognition, 21, 1419-34. doi: $10.1016 /$ j.concog.2012.03.009

Sjoberg, B. M. J., \& Hollister, L. E. (1965). The effects of psychotomimetic drugs on primary suggestibility. Psychopharmacologia, 8, 251-62.

Smilek, D., Dixon, M. J., Cudahy, C., \& Merikle, P. M. (2001). Synaesthetic photisms influence visual perception. Journal of Cognitive Neuroscience, 13, 930-6. doi: $10.1162 / 089892901753165845$

Snowball, A., Tachtsidis, I., Popescu, T., Thompson, J., Delazer, M., Zamarian, L., Zhu, T., \& Cohen Kadosh, R. (2013). Long-term enhancement of brain function and cognition using cognitive training and brain stimulation. Current Biology, 23, 987-992. doi:

10.1016/j.cub.2013.04.045

Spanos, N. P., \& Gorassini, D. R. (1984). Structure of hypnotic test suggestions and attributions of responding involuntarily. Journal of Personality and Social Psychology, 46, 688-96.

Studerus, E. (2013). Psilocybin-induced altered states of consciousness: Tolerability, assessment, and prediction. Saarbrücken, Germany: Südwestdeutscher Verlag für Hochschulschriften.

Studerus, E., Gamma, A., \& Vollenweider, F. X. (2010). Psychometric evaluation of the altered states of consciousness rating scale (OAV). PLoS One, 5, e12412. doi:

10.1371/journal.pone. 0012412 
Terhune, Luke, \& Cohen Kadosh, 2017, chapter in Sensory Blending: On synaesthesia and other phenomena

Szechtman, H., Woody, E., Bowers, K.S., \& Nahmias, C. (1998). Where the imaginal appears real: A positron emission tomography study of auditory hallucinations. Proceedings of the National Academy of Sciences of the United States of America, 95, 1956-1960.

Tart, C. T. (1975). States of consciousness. New York: E. P. Dutton.

Tellegen, A., \& Atkinson, G. (1974). Openness to absorbing and self-altering experiences ("absorption"), a trait related to hypnotic susceptibility. Journal of Abnormal Psychology, $83,268-77$.

Terhune, D. B., Cardeña, E., \& Lindgren, M. (2010). Disruption of synaesthesia by posthypnotic suggestion: An ERP study. Neuropsychologia, 48, 3360-4. doi: 10.1016/j.neuropsychologia.2010.07.004

Terhune, D. B., Cardeña, E., \& Lindgren, M. (2011a). Differential frontal-parietal phase synchrony during hypnosis as a function of hypnotic suggestibility. Psychophysiology, 48, 1444-7. doi: 10.1111/j.1469-8986.2011.01211.x

Terhune, D. B., Cardeña, E., \& Lindgren, M. (2011b). Dissociated control as a signature of typological variability in high hypnotic suggestibility. Consciousness and Cognition, 20, 727-36. doi: 10.1016/j.concog.2010.11.005

Terhune, D. B., \& Cohen Kadosh, R. (2012a). The emerging neuroscience of hypnosis. Cortex, 48, 382-6. doi: 10.1016/j.cortex.2011.08.007

Terhune, D. B., \& Cohen Kadosh, R. (2012b). Posthypnotic manipulation of the visuospatial phenomenology of colour photisms modulates selective attention in synaesthesia. Poster presented at the Association for the Scientific Study of Consciousness, Brighton, UK.

Terhune, D.B., Luke, D.P., Kaelen, M., Bolstridge, M., Feilding, A., Nutt, D., Carhart-Harris, R., \& Ward, J. (2016). A placebo-controlled investigation of synaesthesia-like experiences 
Terhune, Luke, \& Cohen Kadosh, 2017, chapter in Sensory Blending: On synaesthesia and other phenomena

under LSD. Neuropsychologia, 88, 28-34. doi: 10.1016/j.neuropsychologia.2016.04.005

Terhune, D.B., Murray, E., Near, J., Stagg, C.J., Cowey, A., and Cohen Kadosh, R. (2015).

Phosphene perception relates to visual cortex glutamate levels and covaries with atypical visuospatial awareness. Cerebral Cortex, 25, 4341-4350. doi: 10.1093/cercor/bhv015

Terhune, D. B., Rothen, N., \& Cohen Kadosh, R. (2013). Correcting misconceptions about synaesthesia. Neurobiology of Learning and Memory, 103, 1-2. doi:

10.1016/j.nlm.2013.02.004

Terhune, D.B., Song, S.M., Duta, M.D., \& Cohen Kadosh, R. (2014). Probing the neurochemical basis of synaesthesia using psychophysics. Frontiers in Human Neuroscience 8, 89. doi: $10.3389 /$ fnhum. 2014.00089

Terhune, D.B., Tai, S., Cowey, A., Popescu, T., \& Cohen Kadosh, R. (2011). Enhanced cortical excitability in grapheme-color synesthesia and its modulation. Current Biology, 21, 20062009. doi: $10.1016 /$ j.cub.2011.10.032

van Leeuwen, T.M., den Ouden, H.E., \& Hagoort, P. (2011). Effective connectivity determines the nature of subjective experience in grapheme-color synesthesia. Journal of Neuroscience, 31, 9879-9884. doi: 10.1523/JNEUROSCI.0569-11.2011

Wallach, J. V. (2009). Endogenous hallucinogens as ligands of the trace amine receptors: a possible role in sensory perception. Medical Hypotheses 72 (1), 91-94.

Ward, J. (2013). Synesthesia. Annual Review of Psychology, 64, 49-75. doi: 10.1146/annurevpsych-113011-143840

Ward, J., Jonas, C., Dienes, Z., \& Seth, A. (2010). Grapheme-colour synaesthesia improves detection of embedded shapes, but without pre-attentive 'pop-out' of synaesthetic colour. Proceedings of the Royal Society of London. Series B: Biological Sciences, 277, 1021-6. 
Ward, J., Li, R., Salih, S., \& Sagiv, N. (2007). Varieties of grapheme-colour synaesthesia: A new theory of phenomenological and behavioural differences. Consciousness and Cognition, $16,913-31$.

Ward, J., \& Mattingley, J. B. (2006). Synaesthesia: An overview of contemporary findings and controversies. Cortex, 42, 129-36.

Ward, J., \& Wright, T. (2012). Sensory substitution as an artificially acquired synaesthesia. Neuroscience and Biobehavioral Reviews. doi: 10.1016/j.neubiorev.2012.07.007

Wasson, R. G. (1978). The road to Eleusis: Unveiling the secret of the mysteries. New York: Harcourt.

Witthoft, N., \& Winawer, J. (2006). Synesthetic colors determined by having colored refrigerator magnets in childhood. Cortex, 42, 175-83.

Witthoft, N., \& Winawer, J. (2013). Learning, memory, and synesthesia. Psychological Science, 24, 258-65. doi: 10.1177/0956797612452573

Woody, E. Z., \& Barnier, A. J. (2008). Hypnosis scales for the twenty-first century: What do we need and how should be use them? In M. R. Nash \& A. Barnier (Eds.), Oxford handbook of hypnosis (pp. 255-81). Oxford, UK: Oxford University Press.

Yaro, C., \& Ward, J. (2007). Searching for Shereshevskii: What is superior about the memory of synaesthetes? Quarterly Journal of Experimental Psychology, 60, 681-95.

Zeki, S. (1980). The representation of colours in the cerebral cortex. Nature, 284, 412-18. 